\title{
Shared and divergent pathways for flower abscission are triggered by gibberellic acid and carbon starvation in seedless Vitis vinifera $\mathrm{L}$
}

Sara Domingos ${ }^{1,2}$, Joana Fino ${ }^{2,3}$, Vânia Cardoso ${ }^{2}$, Claudia Sánchez ${ }^{4}$, José C. Ramalho ${ }^{1,2,5}$, Roberto Larcher ${ }^{6}$, Octávio S. Paulo ${ }^{3}$, Cristina M. Oliveira ${ }^{1}$ and Luis F. Goulao ${ }^{2,7^{*}}$

\begin{abstract}
Background: Abscission is a highly coordinated developmental process by which plants control vegetative and reproductive organs load. Aiming at get new insights on flower abscission regulation, changes in the global transcriptome, metabolome and physiology were analyzed in 'Thompson Seedless' grapevine (Vitis vinifera L.) inflorescences, using gibberellic acid (GAc) spraying and shading as abscission stimuli, applied at bloom.
\end{abstract}

Results: Natural flower drop rates increased from $63.1 \%$ in non-treated vines to $83 \%$ and $99 \%$ in response to GAc and shade treatments, respectively. Both treatments had a broad effect on inflorescences metabolism. Specific impacts from shade included photosynthesis inhibition, associated nutritional stress, carbon/nitrogen imbalance and cell division repression, whereas GAc spraying induced energetic metabolism simultaneously with induction of nucleotide biosynthesis and carbon metabolism, therefore, disclosing alternative mechanisms to regulate abscission. Regarding secondary metabolism, changes in flavonoid metabolism were the most represented metabolic pathways in the samples collected following GAc treatment while phenylpropanoid and stilbenoid related pathways were predominantly affected in the inflorescences by the shade treatment. However, both GAc and shade treated inflorescences revealed also shared pathways, that involved the regulation of putrescine catabolism, the repression of gibberellin biosynthesis, the induction of auxin biosynthesis and the activation of ethylene signaling pathways and antioxidant mechanisms, although often the quantitative changes occurred on specific transcripts and metabolites of the pathways.

Conclusions: Globally, the results suggest that chemical and environmental cues induced contrasting effects on inflorescence metabolism, triggering flower abscission by different mechanisms and pinpointing the participation of novel abscission regulators. Grapevine showed to be considered a valid model to study molecular pathways of flower abscission competence acquisition, noticeably responding to independent stimuli.

Keywords: Flower shedding, Gibberellin, Grapevine, Light reduction, Metabolomics, RNA-Seq

\footnotetext{
* Correspondence: goulao@reitoria.ulisboa.pt

${ }^{2}$ Instituto de Investigação Científica Tropical, I.P. (IICT), Lisbon, Portugal

7 Present address: Colégio Food, Farming and Forestry, Universidade de

Lisboa (ULisboa), Lisbon, Portugal

Full list of author information is available at the end of the article
} 


\section{Background}

Abscission is the developmental mechanism by which plants are able to shed damaged and excessively formed organs, regulating the metabolic energy required to successfully attain the formation of vegetative and reproductive structures [1]. Abscission encompasses a complex but precise regulation of cell separation that occurs in a specific layer of specialized cells known as abscission zone (AZ) and is simultaneously activated by and responsive to endogenous and exogenous signals, such as abiotic and biotic interactions or exposure to chemical molecules $[2,3]$. Once the AZ is properly differentiated, AZ cells acquire competence to respond to triggering-abscission signals through hormone-mediated pathways. After the activation phase, by modulating the expression of genes involved, among others, in cell wall (CW) remodeling and protein metabolism, and a high number of transcription factors, cell separation and differentiation of a protective layer on the proximal side after organ detachment advance as last steps of the abscission process $[4,5]$. According to the currently accepted model, the endogenous flow level of inhibitory auxin in an organ destined to abscise must drop to acquire sensitivity to ethylene $[6,7]$. Abscisic acid (ABA) is involved by acting as modulator of 1aminocyclopropane-1-carboxylic acid (ACC) levels, and therefore of ethylene biosynthesis [8]. Increased ethylene biosynthesis is associated with the final events of abscission activation, namely by promoting CW disassemblyrelated genes transcription [9, 10]. Increased levels of reactive oxygen species (ROS) have a pivotal role in organ abscission control, encompassing multiple steps of signaling, downstream from ethylene, and associated with ROS-sugar-hormone cross talk [11-14].

In reproductive organs, abscission is also related to lower carbohydrate and polyamine (PA) availability to developing flowers and fruits [15-18]. Together with its role as energy source, glucose acts as a repressing signal of programmed cell death (PCD) [19]. A glucose gradient in the $\mathrm{AZ}$ was recently suggested, similar to the auxin flux that regulates ethylene signaling [2]. In addition, the inflorescence deficient in abscission (IDA) peptide signals and interacting receptor-like-kinases, HAESA and HAESAlike2, were showed to activate mitogen-activated protein kinase (MAPK) cascades leading to the abscission of floral organs in Arabidopsis thaliana L. [20, 21], in a signaling system that was proposed to be conserved and regulate cell separation in other plant species [22].

Strategies that stimulate flower and fruit abscission are widespread horticultural practices, collectively known as thinning. In seedless table grape (Vitis vinifera L.) production, reduction of the number of berries per bunch is mandatory to guarantee bunch quality and decrease fungal diseases incidence [23]. Gibberellic acid (GAc) spraying during bloom, often followed by hand adjustments, is the most common method for thinning in grapevine [23-27], although the mechanisms by which GAc induces abscission remains largely unknown. Gibberellin (GA) perception and signaling investigated in model plants [28] disclosed early recognition via the GA INSENSITIVE DWARF1 (GID1) receptor and interaction between GA-GID complex and DELLA transcription factor responsible for GA signaling repression. Binding of GAGID1 to DELLA induces recognition of DELLA for ubiquitination by a specific F-box protein (GID2) that results in a rapid degradation of DELLAs via the ubiquitin-proteasome pathway. Recently, GA-induced changes in the transcriptome of pre-bloom inflorescences and of berry enlargement stages in grapevine were investigated [29,30] and the results suggested that GAc application to grape flowers and berries has a fairly comprehensive impact on their metabolism mediated by hormone biosynthesis and signaling, in particular through a negative feedback regulation of GAs biosynthesis and signaling $[29,30]$.

Flower abscission can also be boosted by shading conditions (70-90 \% light interception) during bloom $[12,31,32]$, paving the way to explore light management as an alternative thinning method. The pronounced reduction of net photosynthetic rates under shading promotes the competition for photoassimilates between vegetative and reproductive organs, leading to shedding of the later with less sink strength at this early stage of development [33]. Shade-induced changes in the transcriptome of apple (Malus $\times$ domestica) revealed that photosynthesis repression and associated nutrient stress is perceived at the fruit level, its growth is inhibited by a sugar transport blockage, resulting in a decreased auxin transport to the $\mathrm{AZ}$ and concomitant increased sensitivity to ethylene, leading to fruit abscission [18].

Therefore, abscission is a challenging biological question that can be induced by at least two distinct stimuli with distinct physiological basis. Recently, using an experimental assay with potted seeded vines managed under a greenhouse hydroponic production system, and thinned with GAc spraying or via shade nets to reduce intercepted light, we established an efficient method to produce sample sets with predictable abscising potential triggered by different (chemical and environmental) cues, which allowed us to disclose the participation of different metabolic pathways according to the imposed treatment in flower abscission regulation [12]. We now report the effect of the same abscission-inducers using a different genetic background under field conditions. The rationale was that, by using a seedless variety deprived of the main endogenous source of bioactive GAs [34] and developed while adapting to field multiple stresses, the major signals for abscission triggering would be perceived, providing new insights on this subject. Hence, comprehensive cutting- 
edge metabolomics, RNA-Seq transcriptomics and physiological measurements, were performed to allow discussing how environmental (C-shortage) and GAc application act to trigger flower abscission, to identify routes linking the aptitude of an organ to become competent for cell separation and specificities and communication between different pathways leading to organ drop. In addition, the present study provides the first sequential transcriptomic atlas of GAc-induced flower abscission.

\section{Methods}

\section{Experimental conditions and sample collection}

The trail was conducted in a commercial table grape

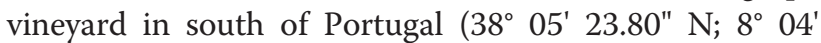
52.7 1" W), using seven-year-old 'Thompson Seedless' (Vitis vinifera L.) vines grafted on '140 Ruggeri' rootstock, spaced $3 \times 3 \mathrm{~m}$, grown under an overhead trellis system covered with plastic, and managed following standard fertilization, irrigation, and pest-management practices. Permission to access and sample at the vineyard was previous agreed under the frame of joint research partnership.

The imposed treatments were: thinning via reduction of intercepted light and chemical thinning with GAc, in five vines per treatment. An additional group of five vines remained untreated to be used as control. Shade was imposed at $50 \%$ cap fall (stage 65 of the $\mathrm{BBCH}$ scale [35]) by covering the vines with polypropylene shading nets (Hubel, Portugal) that intercept $100 \%$ of the incident photosynthetic photon flux density (PPFD), for a period of fourteen days. Chemical treatment consisted in spraying GAc solution (Berelex with $9 \%$ of GAc, Kenogard) at $10 \mathrm{ppm}, 12.5 \mathrm{ppm}$ and $12.5 \mathrm{ppm}$, applied sequentially at $20 \%, 50 \%$ and $100 \%$ cap fall (stages 62,65 and 69 of the $\mathrm{BBCH}$ scale, respectively).

Climate conditions during the assay were monitored above the canopy of shaded and control vines (WatchDog MicroStation, Spectrum Tech., USA) (Additional file 1). Grape inflorescence samples were collected in a time-course assay, at three time-points: 5,7 and 10 days after $100 \%$ cap fall (referred to as $5 \mathrm{~d}, 7 \mathrm{~d}$ and $10 \mathrm{~d}$ ) (Fig. 1). In each point, three independent biological replicates were collected per treatment in the corresponding five-vine plot. Each biological replicate is composed essentially by the flowers with their pedicels from an inflorescence deprived from rachis, immediately frozen in liquid nitrogen, and subsequently fine-powdered and stored at $-80{ }^{\circ} \mathrm{C}$ until use.

\section{RNA deep sequencing and bioinformatic analysis}

Total RNA was extracted and purified from ca. $100 \mathrm{mg}$ frozen inflorescences from each 5 and $7 \mathrm{~d}$ biological sample, using the RNeasy Plant RNA Extraction Kit and RNase-Free DNase Set (Qiagen, Hilden, Germany) following the manufacturer's instructions, but replacing the extraction solution for a $100 \mathrm{mM}$ Tris- $\mathrm{HCl}, 2 \%(\mathrm{w} / \mathrm{v})$ CTAB, $25 \mathrm{mM}$ EDTA and $2 \mathrm{M} \mathrm{NaCl}$ buffer [36]. When traces of contaminant genomic DNA were detected after standard PCR amplification of the ACTIN 1 (ACT1) gene (XM_002282480.3), samples were further digested with RNase-free DNase I (Ambion, Life Techonologies, CA, USA). RNA integrity and purity were evaluated by visual inspection of ribosomal bands in $1.5 \%$ agarose gel electrophoresis and by 2100 Bioanalyzer (Agilent Technologies, CA, USA) readings. Poly(A) mRNA isolation, cDNA synthesis, library generation, indexing, cluster generation and RNA-Seq analyses by Illumina HiSeq 2000 RNA sequencing of $100 \mathrm{bp}$ paired-end reads was carried out by LGC Genomics (Berlin, Germany), using commercial services.

The raw Illumina 100-bp pair-end sequences were deposited in the NCBI Sequence Read Archive (SRA). The reads were quality trimmed using Trimmomatic version 0.32 [37], and surveyed for the presence of rRNA contamination using homology searches against rRNA databases [38]. Alignment against the Vitis vinifera reference genome [39] was then performed with the software Tophat2 version 2.0.12 [40] set with the parameters -D 15 -R 2 -L 22 -i S,1,1.15 and end-to-end mode. Quantification and normalization of gene expression values by Fragments Per Kilobase Of Exon Per Million Fragments Mapped (FPKM) was calculated by Cufflinks version 2.2.1 [41]. Differential expression calculations were handled by DESeq2 version 1.4.5 [42] considering estimation of size factors, a false discovery rate (FDR) of 0.05 and a $-1.5 \geq$ $\log 2$ fold-change $\geq 1.5$, using the raw read counts.

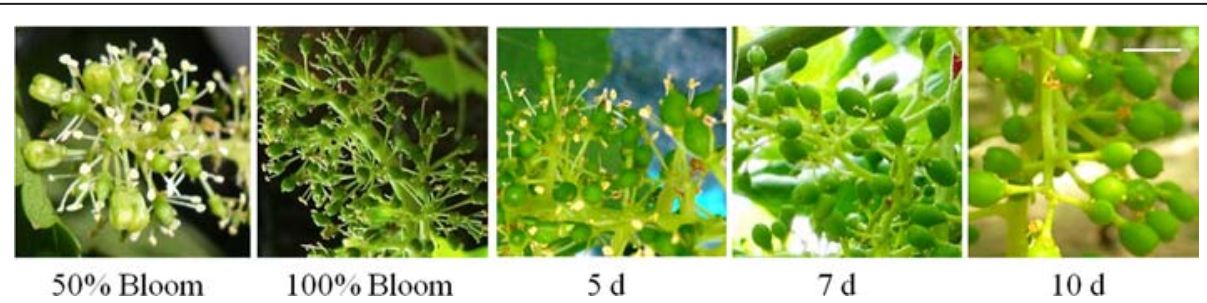

Fig. 1 Aspect of 'Thompson Seedless' inflorescences from $50 \%$ cap fall to 10 days after $100 \%$ cap fall. Samples were collected at 5, 7 and 10 days after $100 \%$ cap fall $(5,7$ and $10 \mathrm{~d})$. Scale bar corresponds to $0.6 \mathrm{~cm}$ 
EuKaryotic Orthologous Groups (KOG) [43], Gene Ontology (GO) and Kyoto Encyclopedia of Genes and Genomes (KEGG) [44] functional annotations were based on sequence homologies against public databases. Rapsearch2 [45] with an e-value cut-off of $10^{-5}$ was used to search against Arabidopsis thaliana sequences in the KOG database and non-redundant ("nr") peptide database (ftp:// $\mathrm{ftp}$.ncbi.nlm.nih.gov/blast/db/ downloaded at November 26, 2013, including all "nr" GenBank CDS translations + PDB + SwissProt + PIR + PRF). To GO and KEGG annotations, the output was submitted to an in-house developed script - Rapsearch2XML (https://github.com/Nymeria8/ Rapsearch2Xml) and then to Blast2GO [46]. GO enriched categories were identified using the $\mathrm{R}$ bioconductor package topGO version 2.18.0 [47], using a Fisher's exact test and a $p$-value $\leq 0.01$.

\section{Data validation by gene expression quantification and correlation between replicates analyses}

Aliquots (150 ng) of the same RNA samples extracted as per 2.1 were used for first-strand cDNA synthesis by M-MLV Reverse Transcriptase (Invitrogen), according to the manufacturer's instructions. The expression of eight genes with significant differences on RNA-seq analysis, involved in auxin and ethylene signaling pathways $[9,48]$ and mitogen-activared protein kinase cascades [20] putatively related to flower abscission regulation, was assessed by q-rtPCR. Their specific primer sequences and properties are given in Additional file 2. qRT-PCR amplifications were conducted in a qTOWER 2.0 (Analytikjena, Germany) thermal cycler in $15 \mu \mathrm{L}$ reactions containing $1 \times$ SsoAdvanced $^{\text {TM }}$ SYBR $^{\circ}$ Green Supermix (Bio-Rad), $0.3 \mu \mathrm{M}$ each primer and $90 \mathrm{ng} \mathrm{cDNA}$.

The amplification cycling profile was: $95{ }^{\circ} \mathrm{C}$ during $30 \mathrm{~s}$; then 40 cycles at $95{ }^{\circ} \mathrm{C}$ for $5 \mathrm{~s}$ and $60{ }^{\circ} \mathrm{C}$ for $30 \mathrm{~s}$. Melting curves were generated to confirm amplification of single products and absence of primer dimerization. For each primer pair, PCR amplification efficiencies were calculated via a calibration dilution curve and slope calculation, using the equation $\mathrm{E}(\%)=\left(10^{[-1 / \text { slope }]}\right) \times 100$ [49]. Data normalization was conducted based on quantification threshold cycle $(\mathrm{Ct})$ values with respect to the geometric average of the $\mathrm{Ct}$ of 3 reference genes [50], polyubiquitin (XM_002282083.2), actin (XM_002282480.3) and glyceraldehyde-3-phosphate dehydrogenase (XM_ 002263109.2). Each analysis was performed in duplicate technical reactions, in each of the three biologic replicates per treatment and condition. To obtain measurements of the correlation between RNA-seq and qRT-PCR data, linear regression and determination coefficient $\left(R^{2}\right)$ were determined between the two methods obtained $\log _{2}$ foldchanges for the same eight genes.

To further investigate the robustness of our RNA-seq dataset, similarity of expression profiles between the three biological replicates was determined by Pearson correlation coefficient (PCC) analyses with R 3.1.2 software using natural logarithm (ln)-transformed read counts for the differentially expressed genes (DEG) as input.

\section{Global and targeted metabolomic profiling}

Circa $200 \mathrm{mg}$ of powdered material from each of the three biological replicates collected at $5 \mathrm{~d}$ and $7 \mathrm{~d}$ for each treatment were lyophilized, extracted with methanol and analyzed using the integrated platform developed by Metabolon ${ }^{\circledR}$ (Durham, USA) consisting of a combination of three independent approaches: ultrahigh performance liquid chromatography/tandem mass spectrometry (UHLC/MS/MS2) optimized for basic species, UHLC/ MS/MS2 optimized for acidic species, and gas chromatography/mass spectrometry (GC/MS). Methods were performed as previously described [51-53]. For UHPLC/MS/ MS2 analysis, aliquots were separated using a Waters Acquity UHPLC (Massachusetts, USA) and analyzed using a LTQ linear ion trap mass spectrometer (Thermo Fisher Scientific Inc., Massachusetts, USA). Each extract was monitored for positive or negative ions in independent injections using separate acid/base dedicated $2.1 \mathrm{~mm} \times$ $100 \mathrm{~mm}$ Waters BEH C18 $1.7 \mu \mathrm{m}$ particle columns, heated to $40{ }^{\circ} \mathrm{C}$. The $\mathrm{MS}$ interface capillary was maintained at $350{ }^{\circ} \mathrm{C}$. The spray voltage for the positive ion injection was $4.5 \mathrm{kV}$, and $3.75 \mathrm{kV}$ for the negative ion injection. The instrument scanned $99-1000 \mathrm{~m} / \mathrm{z}$ and alternated between MS and MS/MS using dynamic exclusion with approximately 6 scans per second. MS/MS normalized collision energy was set to 40 , activation Q 0.25 , and activation time $30 \mathrm{~ms}$, with a $3 \mathrm{~m} / \mathrm{z}$ isolation window. MS/MS scans were collected using dynamic exclusion with an exclusion time of $3.5 \mathrm{~s}$. Derivatized samples for GC/MS were separated on a $5 \%$ phenyldimethyl silicone column with helium as the carrier gas and a temperature ramp from $40{ }^{\circ} \mathrm{C}$ to $300{ }^{\circ} \mathrm{C}$ and then analyzed on a Thermo-Finnigan Trace DSQ MS (Thermo Fisher Scientific Inc., Massachusetts, USA) operated at unit mass resolving power with electron impact ionization and a 50-750 atomic mass unit scan range.

Metabolites were identified by automated comparison of the ion features in the experimental samples to a reference library of chemical standard entries that included retention time, molecular weight $(\mathrm{m} / \mathrm{z})$, preferred adducts, and in-source fragments as well as associated MS/MS2 spectra (Additional file 3) and curated by visual inspection for quality control using a software developed at Metabolon Inc [53]. Raw area counts for each biochemical compound were rescaled by dividing each sample's value by the median value for the specific biochemical. Welch's two-sample t-tests were then used to determine whether or not each metabolite had significantly increased or decreased in abundance using Array Studio software (Omicsoft) and Microsoft Excel ${ }^{\circledR}$ spreadsheets. Mapping of 
metabolites was performed onto general biochemical pathways, as provided in the Kyoto Encyclopedia of Genes and Genomes (KEGG) (www.genome.jp/kegg/) and Plant Metabolic Network (PMN) (www.plantcyc.org/).

Hormone (indole-3-acetic acid (IAA), abscisic acid (ABA), $\mathrm{GA}_{1}, \mathrm{GA}_{4}, \mathrm{GA}_{8}, \mathrm{GA}_{9}, \mathrm{GA}_{12}, \mathrm{GA}_{20}, \mathrm{GA}_{34}, \mathrm{GA}_{53}$ ) extraction and quantification were performed [54] in $5 \mathrm{~d}$, $7 \mathrm{~d}$ and $10 \mathrm{~d}$ inflorescence samples. Starting from lyophilized ca. $300 \mathrm{mg}$ weighed aliquots per sample, $15 \mu \mathrm{L}$ samples were injected on an Acquity UPLC BEH C18 column $(1.7 \mu \mathrm{m}$ film thickness, $2.1 \mathrm{~mm} \times 100 \mathrm{~mm}$; Waters) mounted into an Acquity UPLC Waters equipped with a Xevo TQ MS mass spectrometer (Waters Corporation, Milford, USA). Flow rate was set at $0.45 \mathrm{mLmin}^{-1}$ and column temperature at $40{ }^{\circ} \mathrm{C}$. Eluent $\mathrm{A}$ was a $0.1 \%$ formic acid in a $2 \mathrm{mM}$ ammonium acetate solution and eluent B was methanol with $0.1 \%$ formic acid in a $2 \mathrm{mM}$ ammonium acetate solution. Chromatographic separation was obtained using the following gradient for solvent B: $2 \%$ for $0.5 \mathrm{~min}$, raised to $95 \%$ in $7.25 \mathrm{~min}$, then held at $95 \%$ for $1 \mathrm{~min}$, and back to $2 \%$ in $0.01 \mathrm{~min}$. Column reconditioning was performed holding $\mathrm{B}$ at $2 \%$ per $3 \mathrm{~min}$ before each injection. The transitions are reported in Additional file 4. Sugar (glucose, sucrose, fructose and stachyose) and free PA (putrescine, spermine, spermidine and cadaverine) contents from inflorescence samples collected at same time points were extracted and quantified by high performance liquid chromatography (HPLC) as previous described by [12]. To access the significance of the differences between treatments, one-way ANOVA and Tukey HSD test at $p$-value $\leq 0.05$ were performed using Statistix 9 software.

\section{Exploratory analysis of transcriptome and metabolome profile}

Data regarding transcript and metabolite quantification was natural logarithm (ln) -transformed for adjustment to normal distribution and verified by histogram plotting, using the $\mathrm{R}$ software before and after the transformation. Principal Coordinate Analysis (PCoA) was conducted based on the pair-wise correlation matrix using the NTsys-PC 2.20e software [55]. The DCENTER module was used to transform the symmetric matrix to scalar product and EIGEN for eigenvalues decomposition to identify orthogonal components of the original matrix modules. The minimum-spanning tree was calculated allowing the visualization of the distances between operational units. $\mathrm{R}$ software was used for Orthogonal Signal Correction Partial Least Squares Discriminant Analysis (O-PLS-DA) and heatmap construction with associated hierarchical clustering. Approximately unbiased and bootstrap probability $p$-values were calculated using pvclust version 1.3.2 [56] with UPGMA method and 1000 bootstrap replications.
Vine physiology and final bunch morphology assessment Flower drop was monitored with resource to non-woven cloth bags positioned around 10 bunches per treatment at full bloom and kept until 10d (days after $100 \%$ cap fall). Shoot length and primary and secondary leaf areas were determined at bloom and 15 after, in six shoots per treatment, following non-destructive methods [57]. Estimated leaf chlorophyll content (SPAD-502 m, Minolta, Japan) was measured twice during the shade period ( 2 and 9d). Leaf gas exchange were measured in the morning period (9:00 am - 11:00 am) using a portable $\mathrm{CO}_{2} / \mathrm{H}_{2} \mathrm{O}$ porometer (CIRAS-1, PPSystems, USA), on eight mature leaves from the central part of the shoots, twice during the shade period ( 8 and 10d) and twice after removal of the shading nets (30 and 43d). At harvest (96d), the same bunches used for flower drop monitoring, were collected and the final number of berries was recorded to calculate the flower drop percentages. Bunch weight, rachis length and bunch compactness (number of berries $\mathrm{cm}^{-1}$ of rachis) were also determined. To access the significance of the differences between treatments, one-way ANOVA and Tukey HSD test were performed as previous described for targeted metabolite analysis.

\section{Results}

Effects of GAc and shade on leaf gas exchanges, vegetative and reproductive organs development

During bloom period, no significant differences on the day/night mean temperature and relative humidity were perceived between treatments which were in average $26 / 15{ }^{\circ} \mathrm{C}$ and $57 / 71 \%$, respectively (Additional file 1 ). Conversely, $100 \%$ PPFD interception was observed in the shaded vines. Leaf net photosynthetic rate $\left(\mathrm{P}_{\mathrm{n}}\right)$, stomatal conductance $\left(g_{s}\right)$, vegetative growth and chlorophyll content were reduced, only under shaded conditions (Table 1). Increased shoot growth was observed in vines submitted to GAc treatments, when compared with shade-treated vines.

GAc and shade treatments resulted in the drop of $887 \pm 74$ and $955 \pm 9$ flowers per inflorescence, respectively, corresponding to $83 \%$ and $99 \%$. These values were significantly higher as compared to the control (natural drop flower) that showed a loss of $569 \pm 81$ flowers, corresponding to $63.1 \%$. Therefore, both GAc and shade imposed treatments significantly induced flower abscission, although with a higher magnitude resulting from light interception, validating our experimental setup. After shade removal, leaf gas exchange rates recovered to values not significantly different from control.

At harvest, the increased flower abscission was translated in a reduced berry number and bunch compactness in both treatments (Table 2). Rachis length and bunch weight and yield were also reduced in bunches from shade-treated plants. 
Table 1 Effect of GAc and shade treatments on physiological measurements during shade period

\begin{tabular}{|c|c|c|c|c|c|c|}
\hline & $P_{n}\left(\mu \mathrm{mol} C \mathrm{CO}_{2} \mathrm{~m}^{-2} \mathrm{~s}^{-1}\right)$ & $g_{s}\left(\mathrm{mmol} \mathrm{H} \mathrm{H}_{2} \mathrm{~m}^{-2} \mathrm{~s}^{-1}\right)$ & $\begin{array}{l}\text { Leaf chlorophyll content } \\
\text { (SPAD units) }\end{array}$ & $\begin{array}{l}\text { Total leaf area growth } \\
\left(\mathrm{m}^{2} \text { vine }^{-1} \text { day }^{-1}\right)\end{array}$ & $\begin{array}{l}\text { Shoot growth } \\
\left(\mathrm{cm} \text { day }^{-1}\right)\end{array}$ & Flower drop (\%) \\
\hline Control & $8.7 \mathrm{a}$ & $83.5 \mathrm{a}$ & $25.6 \mathrm{a}$ & $0.914 \mathrm{a}$ & $2.9 a b$ & $63.1 \mathrm{c}$ \\
\hline GAC & $8.8 \mathrm{a}$ & $83.4 \mathrm{a}$ & $24.2 \mathrm{a}$ & 0.917 a & $3.8 \mathrm{a}$ & $83.0 \mathrm{~b}$ \\
\hline \multirow[t]{2}{*}{ Shade } & $0.0 \mathrm{~b}$ & $7.4 \mathrm{~b}$ & $22.5 b$ & $0.052 b$ & $1.6 \mathrm{~b}$ & $99.0 \mathrm{a}$ \\
\hline & $* * *$ & $* * *$ & $* * *$ & $* * *$ & $* *$ & $* * *$ \\
\hline
\end{tabular}

Net photosynthetic rate $\left(P_{n}\right)$, stomatal conductance $\left(g_{s}\right)$, estimated leaf chlorophyll content, total (primary and secondary) leaf area growth, shoot growth and total percentage of flower drop average values are reported. ${ }^{* *}{ }^{* * *}$ mean that the treatments are significantly different at $p$-value $\leq 0.01$ or $\leq 0.001$ (ANOVA). Within each column, different letters indicate significant differences among treatments according to Tukey's HSD test ( $p$-value $\leq 0.05)$

\section{Transcriptome analysis}

Eighteen RNA-seq 100-bp paired-end read libraries were prepared from poly(A) RNA extracted from grapevine inflorescences and an average of 27 million paired end reads were collected per each library (Table 3 ). Approximately $8 \%$ of the reads were trimmed based on the presence of Illumina adapters or low quality bases. After removing rRNA contamination, clean reads were obtained and the statistics of each sample mapping are showed in Additional file 5. Reads mapping to the genome sequence made up approximately $76.8 \pm 1.8 \%$ of the reads (Table 3 ).

A total of 5581 genes were identified as differentially expressed between control and at least one of the libraries from treated samples (Additional file 6). The abbreviations GAc5d, GAc7d, SH5d and SH7d mean the log2 fold-change between gene relative expression obtained in treated and control inflorescences, from samples collected at 5 and 7 days after $100 \%$ cap fall. As shown in Fig. $2 \mathrm{a}$, the shade treatment was responsible for the highest number of DEG, with 1781 and 5060 genes significantly showing differential expression at 5 and $7 d$, respectively. On the other hand, GAc treatment led to the differential expression of 192 and 173 genes, in $5 \mathrm{~d}$ and $7 \mathrm{~d}$ samples, respectively. According to hierarchical clustering analysis, means of expression values of samples collected in the two time points investigated from each thinning treatment, were significantly clustered together (Fig. 3a). Regarding PCoA, the shade-treated biological replicates were differentiated from GAc and control ones by PC1 in both time points, whereas PC2 separated the $7 d$ GAc-treated biological replicates from the controls (Fig. 3b). These results indicate that, while shade treatment affected significantly the overall transcriptome dynamics both at 5 and $7 \mathrm{~d}$, in GAc, only in the second time sampled the treatment effect was above the biological variation between replicates. The OPLS-DA analysis of the differential expressed genes plotted by the KOG categories showed an overlap of gene functional categories (Additional file 7A). A positive significant correlation was found between the log2 fold-changes from qRT-PCR and RNA-Seq transcriptomic datasets, confirming the reproducibility of RNA-Seq data (Additional file 2). In agreement, the robustness of the generated RNA-Seq dataset was further revealed by a high correlation of the transcriptome profiles among three biological replicates per treatment (Additional file 8).

\section{Metabolome analysis}

Regarding global metabolomic analysis, from the 215 metabolites searched by the global metabolic analyses conducted, a total of 105 changed its relative content in at least one of the conditions ( $p$-value $\leq 0.05$ ) (Additional file 9). For the Fig. 2b, the abbreviations GAc5d, GAc7d, SH5d and SH7d mean the log2 fold-change between metabolite relative content obtained in treated and control inflorescences, collected after 5 and 7 days after $100 \%$ cap fall. In samples from the GAc treatment, 30 and three metabolites changed respectively at 5 and $7 \mathrm{~d}$, while in shaded vines, 50 and 62 metabolites changed in the same time points (Fig. 2b). According to hierarchical clustering, the two time points of each treatment were clustered together and the different treatments were

Table 2 Effect of treatments on gas exchange rates after shade period and bunch quality at harvest

\begin{tabular}{lllllll}
\hline & $\mathrm{Pn}\left(\mu \mathrm{mol} \mathrm{CO} \mathrm{m}^{-2} \mathrm{~s}^{-1}\right)$ & $g_{s}\left(\mathrm{mmol} \mathrm{H}_{2} \mathrm{O} \mathrm{m}^{-2} \mathrm{~s}^{-1}\right)$ & Bunch weight $(\mathrm{g})$ & Number of berries & Rachis length $(\mathrm{cm})$ & Bunch compactness \\
\hline Control & 7.3 & 69.7 & $1479.6 \mathrm{a}$ & $324.2 \mathrm{a}$ & $48.5 \mathrm{a}$ & $6.8 \mathrm{a}$ \\
GAc & 8.6 & 81.8 & $821.8 \mathrm{ab}$ & $168.0 \mathrm{~b}$ & $44.9 \mathrm{a}$ & $3.9 \mathrm{~b}$ \\
Shade & 7.0 & 92.6 & $97.0 \mathrm{~b}$ & $14.8 \mathrm{c}$ & $20.8 \mathrm{~b}$ & $0.7 \mathrm{c}$ \\
& $\mathrm{ns}$ & $\mathrm{ns}$ & $* *$ & $* * *$ & $* * *$ & $* * *$ \\
\hline
\end{tabular}

Net photosynthetic rate $\left(P_{n}\right)$ and stomatal conductance $\left(g_{s}\right)$ after shade period, and bunch weight, number of berries, rachis length and bunch compactness average values are reported. $\mathrm{ns}^{* *},{ }^{* * *}$ mean that the treatments are not significantly different, are significantly different at $p$-value $\leq 0.01$ or $\leq 0.001$ (ANOVA). Within each column, different letters indicate significant differences among treatments according to Tukey's HSD test ( $p$-value $\leq 0.05$ ) 
Table 3 RNA-Seq data overview

\begin{tabular}{llcc}
\hline & Raw read pairs (x1000) & $\begin{array}{l}\text { Remaining reads } \\
\text { after trimming (\%) }\end{array}$ & Mapped reads (\%) \\
\hline C5d & $36342 \pm 5193$ & $91.1 \pm 2.5$ & $76.9 \pm 0.7$ \\
C7d & $24725 \pm 603$ & $92.1 \pm 0.8$ & $76.1 \pm 0.7$ \\
GAc5d & $23705 \pm 1936$ & $93.2 \pm 0.8$ & $77.9 \pm 2.2$ \\
GAc7d & $20957 \pm 1580$ & $91.1 \pm 1.3$ & $76.0 \pm 0.7$ \\
SH5d & $26103 \pm 1920$ & $92.4 \pm 1.0$ & $80.0 \pm 5.5$ \\
SH7d & $30549 \pm 1242$ & $92.2 \pm 1.6$ & $74.1 \pm 1.0$ \\
\hline
\end{tabular}

Reads number obtained in each treatment, percentage of reads after data trimming and of successfully mapped reads after rRNA contamination removal (mean of three independent biological replicates \pm standard error (se))

separated with strong confidence based on bootstrap analyses (Fig. 3c). Figure 3d shows the association between biological replicates from all samples. PC1 separated shade from GAc treatment, while PC2 distinguished control replicates from treated ones, in both time points. According to OPLS-DA, metabolites clustered by super-pathway showed specific distribution patterns (Additional file 7B). Altered metabolites derived from amino acid metabolism are identified as the major source of the variance in our data set. The results also show that component 1 clearly separated changes on metabolites from peptide

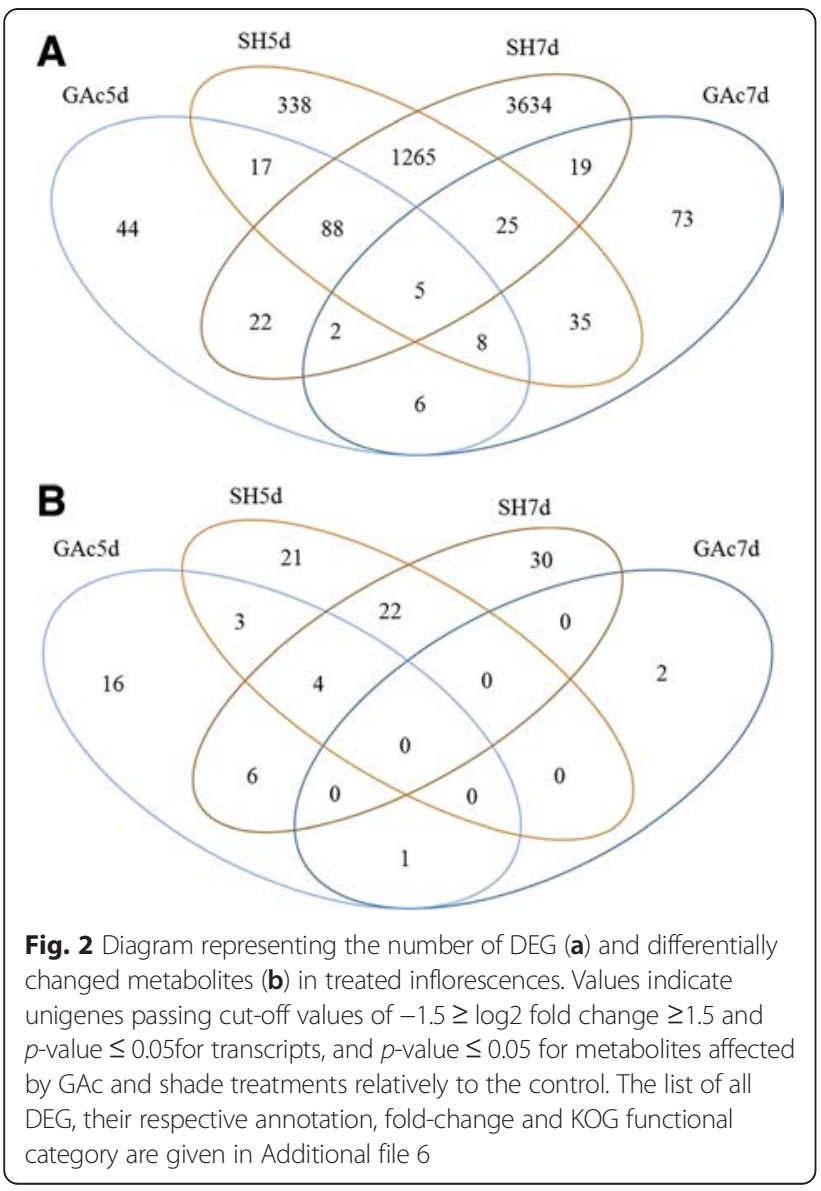

metabolism from secondary metabolites and, to a less extent, from carbohydrates, lipids and nucleotides (Additional file 7B).

\section{Functional annotation and enrichment analysis}

From the total 5581 DEG, 2079 were automatically classified in KOG functional categories, 748 were manually assigned to the same categories according to the similarity with the automatically annotated, 393 were assigned to other functions and 2361 were classified as general or unknown function (Additional file 6). The most representative functional categories in shade-treated samples at both time points investigated were: signal transduction mechanisms, secondary metabolites biosynthesis, transport and catabolism, carbohydrates transport and metabolism, transcription and posttranslational modification, protein turnover, and chaperones (Additional file 10). At the metabolite level, the most representative pathways included amino acid and peptide, carbohydrate, lipid and cofactors metabolism in both time points, whereas secondary metabolism and nucleotide metabolism were most representative only at $5 \mathrm{~d}$ and $7 \mathrm{~d}$, respectively.

To cope with the exploratory analysis results observed at transcriptome level (Fig. 3a), only GAc-treated samples collected at $7 \mathrm{~d}$ will be discussed. In this sample set, energy production and conversion, translation and ribosomal structure, carbohydrates transport and metabolism, transcription and signal transduction mechanism functional categories were the most representative functional categories (Additional file 10). Based on metabolome analysis, carbohydrates, amino acid and peptide, secondary metabolism, nucleotide and cofactor, prosthetic group and electron carrier metabolism were the most representative superpathways at $5 \mathrm{~d}$, while nucleotide, hormone and cofactors metabolisms were the only classes represented at $7 \mathrm{~d}$ in GAc treated samples.

In addition, enzyme identification among DEG and its KEGG metabolic pathway assignment allowed identifying 24 and 205 enzymatic classes and 32 and 113 KEGG pathways for GAc- and shade-abscission inducing treatments, respectively (Additional file 11). The most representative KEGG metabolic pathways were oxidative phosphorylation and purine metabolism in GAc-treated inflorescences, and starch and sucrose metabolism and purine metabolism in shade-treated inflorescences. According to GO enrichment analysis, which demonstrate if a given pathway is predominant in our data set comparing to whole-genome background ( $p$-value $\leq 0.01$, Additional file 12), 460 terms were found to be enriched. Acyclic graphs showing the top 5 and top 5-related GO terms mostly affected in treatment and time point (Additional file 13) suggested that genes related to electron and proton transport, oxidative phosphorylation were enriched in 


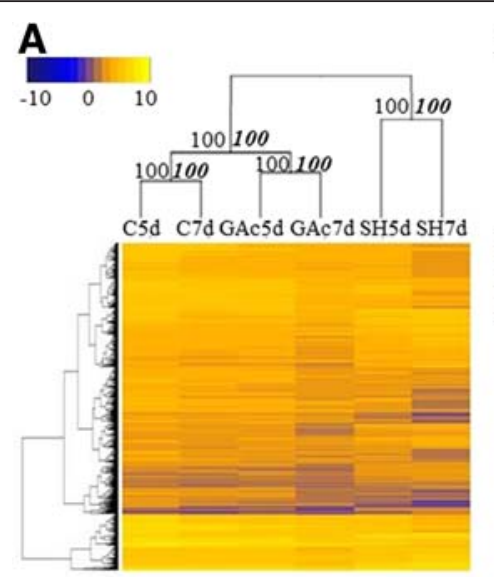

B
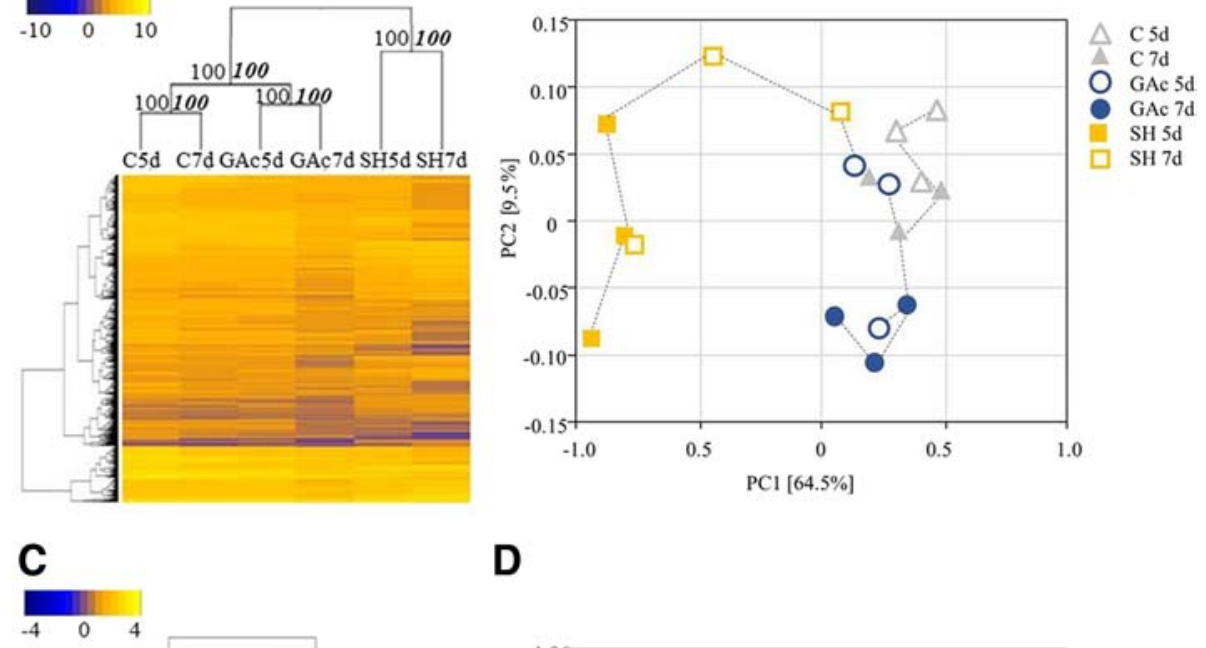

D
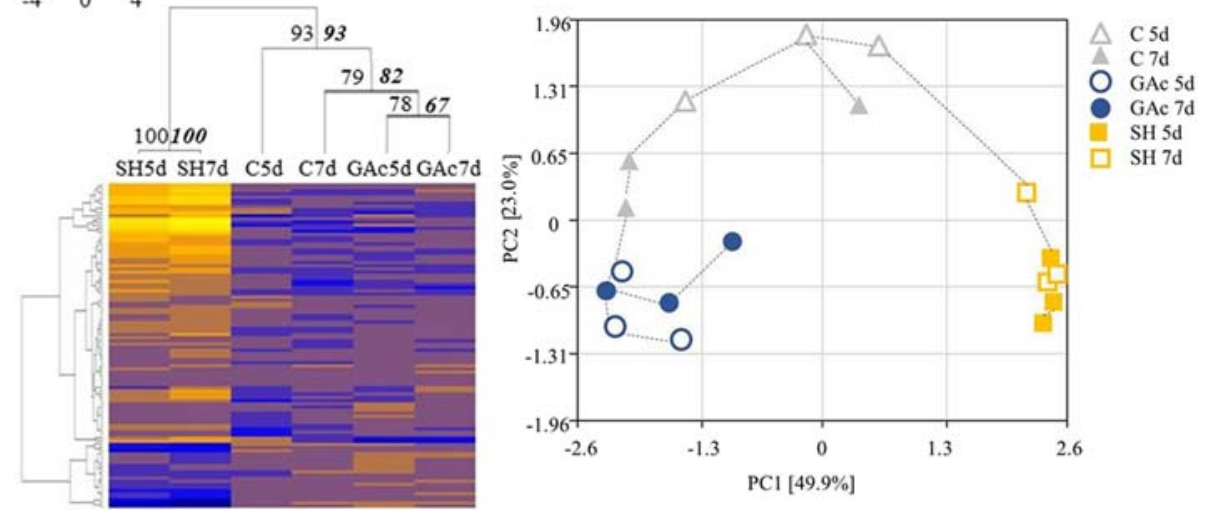

Fig. 3 Hierarchical clustering and principal coordinate analysis (PCOA) of transcriptomic and metabolomic profile. Hierarchical clustering of expression values (a) and metabolite content (c) at different sampled stages. Each column represents the mean value for each treatment at each sampled stage (5 and 7 days after cap fall (d)). Data were In-transformed and yellow tones represent higher values while blue tones represent lower values. The strength of dendrogram nodes was estimated with a bootstrap analysis using 1000 permutations, values represented in the left side of internal nodes are the approximately unbiased $p$-values (AU), bold and italic values on the right side represented the bootstrap probability value. Principal Coordinate Analysis of expression values (b) and metabolite content (d) of control (triangles), GAc (circles) and shade (squares) treated inflorescences, at 5d (open) and $7 \mathrm{~d}$ (close), and respective biological replicates. The variance explained by each coordinate (\%) is given under brackets

GAc-treated inflorescences while genes involved in response to light signal and secondary metabolism were enriched in shade samples, concerning biological processes. Among molecular functions, terms were mostly related to $\mathrm{NADH}$ oxidoreductase and dehydrogenase and rRNA binding in GAc-treated inflorescences, and to oxidoreductase, electron carrier, tetrapyrrole binding, hydrolase, glycosyl transferase and phenylalanine ammonia-lyase activities in shade-treated inflorescences. Regarding cellular components, the most enriched categories induced by GAc treatment were intracellular membrane-bounded organelle, chloroplast and cytoplasm, while apoplast, thylakoid and CW terms were enriched in shade treatment.

\section{Effect of GAc treatment on metabolic pathways}

As shown in Table 4, the specific genes most affected by GAc treatment were all up-regulated. The most representative category was energy production and conversion, comprising genes encoding ATP synthases, cytochrome $c$ biosgenesis protein, cytochrome oxidase, NADH dehydrogenases, an ATPase, and ribosomal proteins.

The most abundant metabolites specifically altered in result of the GAc treatment, were $\beta$-alanine and guanine from nucleotide metabolism, carnitine from cofactor metabolism and mannitol and galactose from carbohydrates metabolism (Fig. 4). Giberellate was only detected in GAc treated samples at both time points, presumably of exogenous origin. Targeted metabolite analysis, allowed detecting increased putrescine and $\mathrm{GA}_{8}$ molecules and to confirm the rise of GAc in GAc-treated inflorescences at $7 \mathrm{~d}$ (Table 5). Cadaverine, IAA, $\mathrm{GA}_{1}, \mathrm{GA}_{4}, \mathrm{GA}_{9}$, $\mathrm{GA}_{12}, \mathrm{GA}_{20}, \mathrm{GA}_{34}, \mathrm{GA}_{53}$ readings were below the detection threshold, so could not be quantified. Spermine, 
Table $\mathbf{4}$ List of top ten DEG specific of GAc treatment

\begin{tabular}{llclll}
\hline Gene ID & GAc5d & GAc7d & Annotation & UniprotKB & Functional category \\
\hline VIT_09s0070g00890 & & 1.96 & Ribosomal protein S7 & F6I3F7 & Translation, rib. struct. and biog. \\
VIT_00s0246g00230 & 1.98 & Cytochrome oxidase subunit III, predicted & F6HML2 & Energy product. and conversion \\
VIT_10s0003g04310 & 2.00 & Vacuolar H +-ATPase V0 sector, subunits c/c' & D7TKE9 & Energy product. and conversion \\
VIT_08s0056g01050 & 2.03 & NADH dehydrogenase subunit 1 (chloroplast) & F6HMW3 & Energy product. and conversion \\
VIT_14s0030g00680 & 2.05 & Ribosomal protein S4, predicted & D7TUX0 & Translation, rib. struct. and biog. \\
VIT_00s0198g00060 & 2.06 & Ribosomal protein S7, predicted & F6I245 & Translation, rib. struct. and biog. \\
VIT_00s0246g00170 & & 2.10 & Cytochrome c biogenesis protein (chloroplast) & F6HMK6 & Energy product. and conversion \\
VIT_00s0854g00040 & 1.50 & 2.11 & NADH dehydrogenase subunit 4 (mitochondrion) & F6HWW5 & Energy product. and conversion \\
VIT_09s0002g00310 & & 2.21 & ATP synthase F0 subunit 6, predicted & D7TZJ7 & Energy product. and conversion \\
VIT_1450036g01270 & 1.55 & 2.44 & ATP synthase F0 subunit 6, predicted & EOCU73 & Energy product. and conversion
\end{tabular}

Gene code identification, fold-change, annotation, UniProtKB accession number and KOG functional category are showed. Data were obtained from 3 independent biological replicates

spermidine, glucose and fructose contents were not different between treated inflorescences and control. Due to the relatively lower number of GAc-induced alterations particularly when compared to those triggered by shade imposition, it was possibly to map it onto simplified metabolic pathways (Fig. 5).

\section{Changes on carbohydrate, cofactor, amino acid and nucleotide metabolism and energy production processes} Glucose-6-phosphate (G6P), fructose-1,6-bisphosphate $(\mathrm{F} 1,6 \mathrm{P} 2)$ and mannose-6-phosphate (M6P), fructose and mannose levels were reduced, while mannitol, which can be synthesized via M6P degradation, and galactose increased in inflorescences from GAc-treated vines. Enhanced photosynthetic and respiratory metabolisms can be hypothesized based on the up-regulation of genes encoding photosystem I and II associated proteins, ribulose-1,5-bisphosphate carboxylase-oxygenase (RuBisCO, EC 4.1.1.39), NADH dehydrogenases (EC 1.6.5.3) and cytochrome-c oxidases (EC 1.9.3.1) and increased glycolate relative content (Additional file 6). Isocitrate relative content decrease and fumarate increase were observed, both associated with the TCA cycle. Cofactors metabolism was also affected, as disclosed by decreased relative contents of nicotianamine and increased nicotinamide and carnitine.

Amino acid and nucleotide pathways were favored in response to GAc treatment comparing to controls, as revealed by increased lysine, isoleucine and polyamine metabolisms and increased pyrimidine and purine metabolisms, respectively (Fig. 5). Conversely $\mathrm{N}$-acetylputrescine levels, involved in putrescine degradation declined. Genes encoding nucleoside-triphosphatase (EC 3.6.1.15), RNA polymerases (EC 2.7.7.6) and $\mathrm{H}^{+}$-translocating ATPase (EC 3.6.3.6) were up-regulated.

\section{Changes on hormone biosynthesis, transcription factors and lipid and secondary metabolism}

A gene encoding an S-beta-glucosyltransferase (EC 2.4.1.195) involved in indole-3-acetic acid (IAA) biosynthesis and secondary metabolism, was up-regulated following GAc treatment.

The down-regulation of a gene encoding a gibberellin 3-beta-dioxygenase (GA3ox) (EC 1.14.11.15) was disclosed and ETHYLENE-RESPONSIVE TRANSCRIPTION FACTOR RAP2-3 (ERF RAP2-3) was the only transcript of hormone signaling pathways affected by GAc (Table 11). The expression of a gene encoding a thioredoxin peroxidase (EC 1.11.1.15) and the relative content of $\beta$ tocopherol, associated to reactive oxygen species (ROS) detoxification mechanism were also affected.

As showed in Fig. 5, among lipid-related pathways, glycerolipid and glycerophospholipid metabolism, fatty acid degradation and linoleic acid metabolism were represented.

Secondary metabolic pathways were also significantly altered with the increase of salidroside, naringenin and quercetion-3-O-glucoside, 2,4,6-trihydroxybenzoate and arbutin contents and down-regulation of genes encoding a peroxidase (EC 1.11.1.7) and a hyoscyamine 6dioxygenase (EC 1.14.11.9), acting in phenylpropanoids, flavonoids and benzenoids biosynthesis and metabolism pathways. Two genes from MYB transcription factors family were down-regulated (Additional file 6).

\section{Effect of shade treatment on metabolic pathways}

Shade imposition resulted in a more pronounced change in the number of genes differentially transcribed and metabolites differentially accumulated than GAc spraying (Tables 4 and 6, Fig. 4). As shown in Table 6, secondary metabolism-related genes encoding a specific MYB transcription factor, flavonol synthase and chalcone synthase, 

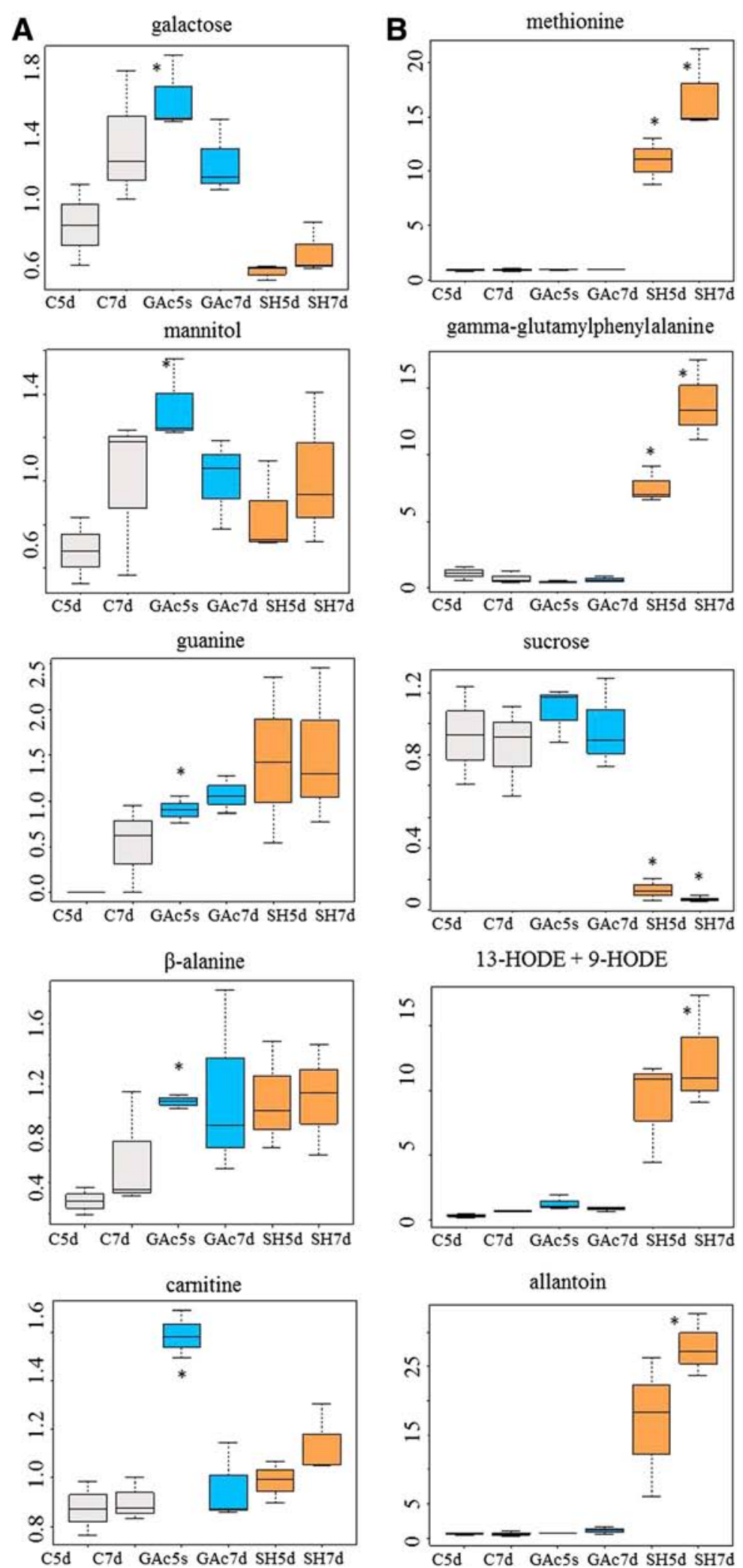

Fig. 4 Relative content evolution of the top five metabolites specific of GAc (a) and shade (b) treatments. Asterisks identify which treatment is different from the control. Data were scale imputed median $=1$. Gray, blue, and orange represent samples from control, GAc and shade treatments, respectively. Data were obtained from 3 independent biological replicates 
Table 5 Changes on metabolite relative content assessed by target chromatography in treated inflorescence comparing to control

\begin{tabular}{|c|c|c|c|c|c|c|c|}
\hline Metabolite & GAc 5d & GAc 7d & GAc 10d & $\mathrm{SH} 5 \mathrm{~d}$ & SH 7d & SH 10d & Super pathway \\
\hline Sucrose & & & & -1.19 & -1.60 & -1.63 & $\overline{\text { Carbohydrate }}$ \\
\hline Putrescine & & 0.52 & & -1.69 & -1.40 & & Polyamines \\
\hline Abscisic acid & & & & -0.94 & -0.71 & & Hormones \\
\hline Gibberellic acid & & 2.36 & & & & & \\
\hline Gibberellin 8 & & 1.81 & & & & & \\
\hline
\end{tabular}

Metabolite, respective fold-change and super pathway are reported. Data were obtained from 3 independent biological replicates

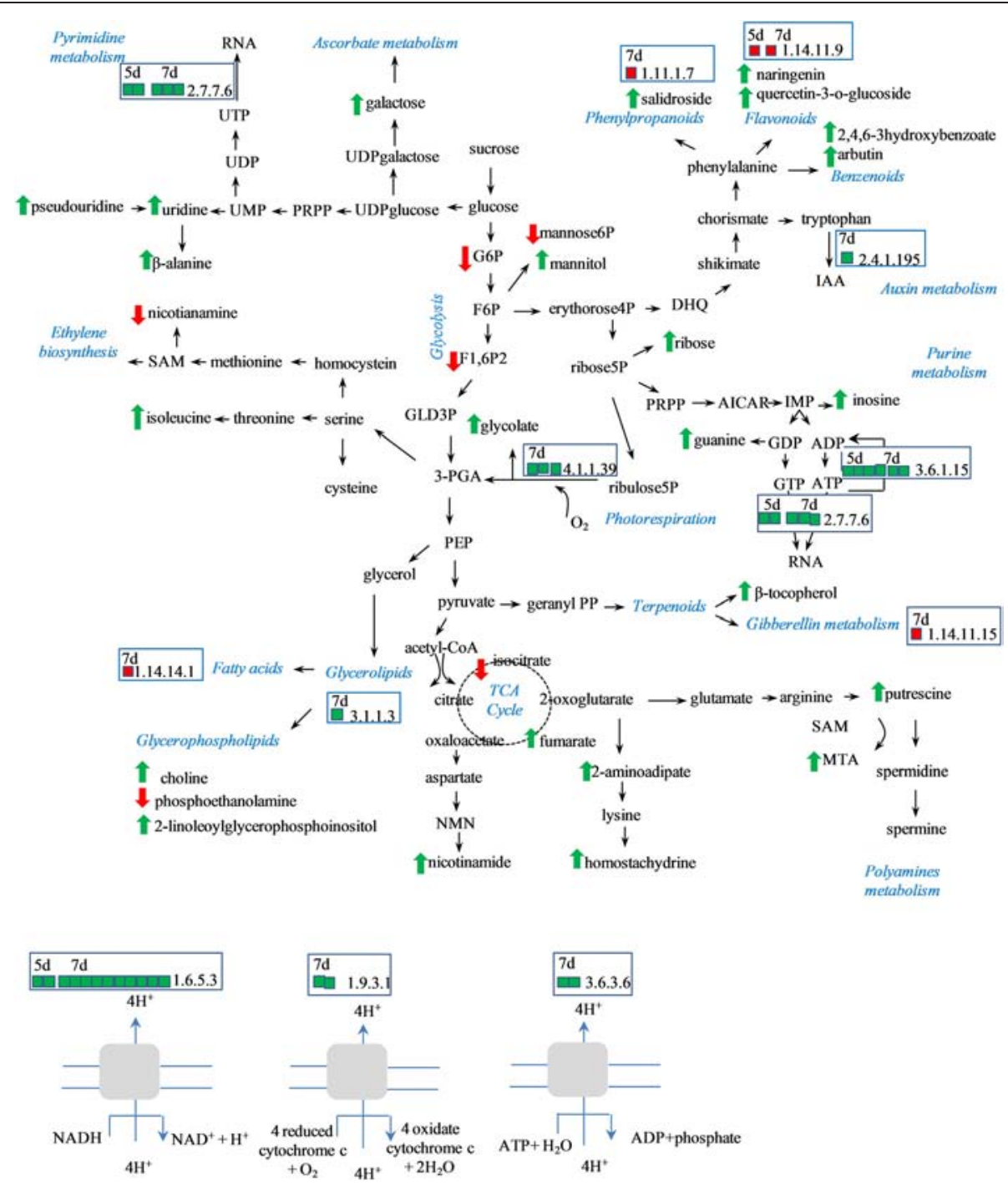

Fig. 5 Changes on transcriptomic and metabolic profiles mapped onto simplified metabolic pathways, observed in GAc-treated inflorescences. Red and green squares represent down and up-regulation of the transcripts, respectively. Gene description and fold-change corresponding to enzyme codes are given in Additional file 11. Red and green arrows represent decreased and increased metabolite accumulation, respectively. Description of enzyme codes: 1.11.1.7 - peroxidase; ec:1.14.11.15 - 3beta-dioxygenase; 1.14.11.9 - 3-dioxygenase; 1.14.14.1 - monooxygenase; 1.6.5.3 - reductase (H + -translocating); 1.9.3.1 - cytochrome-c oxidase; 2.4.1.195 - S-beta-glucosyltransferase; 3.1.1.3 - lipase; 3.6.1.15 - nucleoside-triphosphatase; 3.6.3.6 ATPase; 4.1.1.39 - carboxylase 
Table 6 List of top ten DEG specific of shade treatment

\begin{tabular}{llrlll}
\hline Gene ID & SH5d & SH7d & Annotation & UniprotKB & Functional category \\
\hline VIT_11s0016g01320 & & -6.51 & Transcription factor MYB, predicted & F6HGP6 & Transcription \\
VIT_18s0001g03470 & -3.26 & -5.70 & Flavonol synthase, predicted & F6H0T8 & Secondary metab. bios. transp. cat. \\
VIT_04s0043g00650 & & -5.63 & Cullin-1 isoform 1, predicted & & Cell cycle control, cell div., chrom. part. \\
VIT_14s0068g00930 & -2.50 & -5.32 & Chalcone synthase & & Secondary metab. bios. transp. cat \\
VIT_18s0001g11010 & & 5.42 & Ca'independent phospholipase A2, predicted & F6H017 & Lipid transport and metabolism \\
VIT_13s0019g03070 & 3.31 & 5.46 & Small heat-shock protein Hsp26, predicted & F6HNN6 & Posttranslational mod., protein turn., chap. \\
VIT_05s0020g02170 & 3.99 & 5.73 & Sugar transporter ERD6-like 16-like, predicted & F6HDJ1 & Carbohydrate transport and metabolism \\
VIT_00s0561g00020 & 3.86 & 5.73 & Stem-specific protein TSJT1-like & D7TYY3 & Other \\
VIT_02s0033g00830 & & 5.75 & GTPase Rab11/YPT3, predicted & F6I079 & Intracellular traff., secretion, vesic. transp. \\
VIT_00s0586g00030 & 3.91 & 5.80 & Stem-specific protein TSJT1-like, predicted & D7UE87 & Other
\end{tabular}

Gene code identification, fold-change, annotation, UniProtKB accession number and KOG functional category. Data were obtained from 3 independent biological replicates

genes encoding a cullin protein, a sugar transporter, stem-specific proteins and a small GTPase protein were the most significantly induced genes, specific for the shade treatment.

The most affected metabolites, specifically as result of the shade treatment (Fig. 4b) derived from amino acid and peptide (methionine and gamma-glutamylphenylalanine), carbohydrate (sucrose), lipid (13-HODE + 9HODE) and nucleotide (allantoin) metabolisms. Targeted metabolite analysis confirmed the reduction of putrescine and sucrose contents detected in global metabolomic analysis, and provided additional data of a significant decrease of ABA levels 5 and $7 d$ in inflorescences sampled from shade treated plants.

Changes on amino acid, peptide and nucleotide metabolism Amino acids metabolism was largely affected by shade treatment at the transcriptomic level, inducing alterations in phenylalanine, cysteine, methione, glycine, serine and threonine-related pathways, followed by alanine, aspartate, arginine, glutamate, glutamine, tyrosine, tryptophan, valine, leucine, isoleucine, proline and polyamine related paths. This result also observed regarding changes in metabolite accumulation, which encompass increased abundance of 30 amino acids or amino acidrelated metabolites and reduced shikimate, putrescine and 4-acetamidobutanoate relative contents in shaded inflorescences. Glutathione and $\gamma$-glutamil peptides accumulation was likewise favored in shade treatment (Additional file 6 and Additional file 9).

DEG associated with purine and pyrimidine nucleotides metabolisms were predominantly up-regulated in result of the shade treatment and the same pattern was observed in associated metabolites, except for guanosine and inosine abundance.

\section{Changes on carbohydrate metabolism, transport and signaling pathways}

Carbohydrate-related pathways were mostly repressed in shaded inflorescences, including photosynthesis, chlorophyll metabolism, carbon fixation, glycolysis, pyruvate metabolism, TCA cycle, starch and sucrose metabolism, pentose phosphate pathway, fructose and mannose metabolism, amino sugar and nucleotide sugar metabolism, galactose metabolism, pentose and glucuronate interconversions and inositol phosphate metabolisms. At the metabolomic level, malate, citromalate, 2-ketogulonate, gluconate, xylose, inositol, glucose and sucrose decreased while fumarate, arabonate and xylonate were showed to increase in samples from the shade treatment.

Alterations on sugar signaling pathways and transport were induced by shade treatment during bloom, as displayed in Table 7. Genes encoding sugar metabolizing enzymes such as threalose-6-phosphate synthases, sucrose synthases and invertases showed a global up-regulation pattern. Genes encoding glucose-6-phosphate translocators and sugar transporter SWEET1 and 3 were predominantly down-regulated, whereas genes encoding sugar transporter SWEET2 and 10, putative hexose transporter and sugar transporters ERD6-like, implicated in transport of sugars out of the vacuole in C-starvation conditions [58] were up-regulated.

\section{Changes on hormone metabolism and signaling pathways}

In what concerns hormone metabolism and signaling pathways, genes involved in ethylene and auxin related pathways were highly represented in samples from the thinning by shade treatment (Table 8 ). Genes encoding $\mathrm{S}$-adenosylmethionine synthase (SAM-S) were downregulated while the expression of genes encoding ACC oxidases, ETHYLENE INSENSITIVE 3-LIKE (EIN3) and 
Table 7 DEG involved in sugar signaling and transport in shade-treated inflorescences and respective fold-change

\begin{tabular}{|c|c|c|c|}
\hline Gene family & GID & SH5d & SH7d \\
\hline \multirow[t]{2}{*}{ SnRK1 } & VIT_01s0026g01740 & & -1.61 \\
\hline & VIT_06s0009g01930 & 1.69 & 2.35 \\
\hline \multirow[t]{2}{*}{ HK } & VIT_06s0061g00040 & & 2.14 \\
\hline & VIT_18s0001g14230 & & -2.57 \\
\hline FK & VIT_05s0102g00710 & & -1.60 \\
\hline \multirow[t]{11}{*}{ T6PS } & VIT_00s0173g00110 & 2.03 & \\
\hline & VIT_00s0233g00030 & & 2.13 \\
\hline & VIT_01s0011g05960 & 1.67 & 2.05 \\
\hline & VIT_01s0026g00280 & 2.05 & 2.87 \\
\hline & VIT_02s0012g01680 & & -1.54 \\
\hline & VIT_02s0154g00110 & & 2.40 \\
\hline & VIT_06s0009g01650 & 2.47 & 3.11 \\
\hline & VIT_10s0003g01680 & 1.76 & 2.55 \\
\hline & VIT_10s0003g02160 & & -2.38 \\
\hline & VIT_12s0028g01670 & 1.83 & 2.25 \\
\hline & VIT_17s0000g08010 & 2.92 & 3.83 \\
\hline SUS & VIT_07s0005g00750 & 1.90 & 2.66 \\
\hline \multirow[t]{6}{*}{ INV } & VIT_00s0233g00010 & 1.60 & 2.46 \\
\hline & VIT_00s2527g00010 & & 2.27 \\
\hline & VIT_02s0154g00090 & & 1.63 \\
\hline & VIT_05s0077g00510 & 2.31 & 3.18 \\
\hline & VIT_14s0060g00860 & 1.52 & 2.25 \\
\hline & VIT_16s0022g00670 & & -2.90 \\
\hline INV-I & VIT_18s0075g00280 & -2.05 & \\
\hline \multirow[t]{8}{*}{ G6PT } & VIT_01s0011g00590 & & -2.64 \\
\hline & VIT_06s0004g02710 & -2.64 & -3.46 \\
\hline & VIT_06s0004g07960 & & -1.73 \\
\hline & VIT_10s0116g00760 & & -1.60 \\
\hline & VIT_14s0066g01000 & & -2.13 \\
\hline & VIT_15s0024g01440 & & -2.31 \\
\hline & VIT_17s0000g08560 & & -2.35 \\
\hline & VIT_18s0001g06300 & & 1.72 \\
\hline \multirow[t]{4}{*}{ SWEET } & VIT_16s0050g02540 & & -2.03 \\
\hline & VIT_17s0000g00830 & 1.92 & 1.62 \\
\hline & VIT_18s0001g15330 & -1.60 & -1.92 \\
\hline & VIT_19s0014g00280 & & 2.54 \\
\hline \multirow[t]{2}{*}{ HT } & VIT_00s0181g00010 & 1.83 & 2.60 \\
\hline & VIT_16s0013g01950 & 1.76 & 2.43 \\
\hline \multirow[t]{2}{*}{ ERD6 } & VIT_05s0020g02170 & 3.99 & 5.73 \\
\hline & VIT_07s0104g00830 & & 1.80 \\
\hline
\end{tabular}

SnRK1: serine/threonine-protein kinase SnRK1; HK: hexokinase; FK: fructokinse; T6PS: trehalose-6-phosphate synthase; SUS: sucrose synthase; ÍNV: invertase; INV-I: invertase inhibitor; G6PT: glucose-6-phosphate/phosphate translocator 2; SWEET: bidirectional sugar transporter SWEET; HT: hexose transporter; EDR6: sugar transporter ERD6-like

Up-regulation is marked as green and down-regulation as red background. Data were obtained from 3 independent biological replicates

ERFs showed predominantly an up-regulation. Auxin biosynthetic pathway from thyptophan was favored as suggested by the up-regulation of a tryptophan aminotransferase-related gene. Genes encoding auxin binding proteins $(\mathrm{ABP})$ and transport inhibitor response 1 (TIR1) auxin receptors were up-regulated, while $A u x / I A A$, AUXIN RESPONSIVE FACTOR (ARF) and AUXIN EFFLUX CARRIERS (AEC) were down-regulated. The synthesis of indole-3-acetic acid (IAA)-amino acid conjugates was induced by the up-regulation of GH3.9 gene at $5 \mathrm{~d}$.

The expression of genes encoding gibberellin20oxidase (GA20ox), gibberellin3-beta-dioxygenase (GA3ox) and gibberellin2-oxidase (GA2ox) was also significantly regulated (Table 8). GA signaling pathway was repressed, with a concomitant up-regulation of a DELLA gene and down-regulation of GID2, responsible for DELLA degradation [59].

Genes involved in $\mathrm{CK}$ activation, such as those encoding a UDP-glycosyltransferase 85A1 (EC 2.4.1.215), zeatin Oglucosyltransferase and $\mathrm{CK}$ riboside 5'-monophosphate phosphoribohydrolase were significantly affected by the imposition of the shade treatment. Genes encoding the CK receptors histidine kinases and histidine-containing phosphotransferase, and CK dehydrogenase enzyme, involved in its degradation, were induced. Shade also promoted the upregulation of genes involved in brassinosteroids (BR) signal transduction. In addition, the expression of genes encoding cyclin-D3 (CYCD3) proteins, which are downstream components of the $\mathrm{CK}$ and BR-signaling pathways that promotes cell division [60], was significantly downregulated, and a SENESCENCE RELATED GENE (SRG1) was up-regulated, in inflorescences from shaded vines, at 7d (Additional file 6).

Genes encoding ABA synthesis and degradation enzymes, such as aldehyde oxidase and abscisic acid 8'hydroxylase, respectively, were up-regulated. These changes on ABA metabolism were also verified as decreased ABA relative content in shaded inflorescences (Table 4). In the ABA-signal transduction pathway, down-regulation of protein phosphatase $2 \mathrm{C}$, which is a negative regulator of $\mathrm{ABA}$ response and up-regulation of SnRK2 were observed, suggesting a de-repression of ABA signaling in shaded inflorescences.

The expression of genes encoding methyltransferase enzymes responsible for conversion of jasmonic (JA) and salicylic acids (SA) in methyljasmonate and methylsalicylate, respectively, was down-regulated. JA-mediated signaling pathway was also affected, as revealed by the up-regulation of a gene encoding TIFY9 which negatively regulates a key transcriptional activator of jasmonate responses [61].

\section{Changes on lipid, cofactor and secondary metabolism}

Impact on lipid-related pathways was disclosed as glycerolipid, glycerophospholipid and sphingolipid metabolism, fatty acid biosynthesis, elongation and degradation, linoleic and arachidonic acid metabolism, unsaturated fatty acids biosynthesis, alkaloid biosynthesis, ether lipid metabolism and cutin, suberine and wax biosynthesis were affected in shade-treated inflorescences (Additional file 11). In particular, genes encoding lipoxygenase (EC 1.13.11.12) and lipase (EC 3.1.1.3) enzymes were highly represented and mostly up-regulated. At the metabolite level, a global increase of fatty acids, oxylipins (HODE), glycerolipids, sterols and glycerophospholipids was also verified (Additional file 9). 
Table 8 DEG involved in hormone biosynthesis, metabolism and signaling pathways in shade-treated inflorescences and respective fold-change

\begin{tabular}{|c|c|c|c|c|c|c|c|c|c|}
\hline & $\begin{array}{c}\text { Gene } \\
\text { family }\end{array}$ & GID & SH5d & SH7d & & $\begin{array}{c}\text { Gene } \\
\text { family }\end{array}$ & GID & SH5d & SH7d \\
\hline \multirow{6}{*}{$\overline{\mathbf{A B A}}$} & $\mathrm{AO}$ & $\begin{array}{l}\text { VIT_18s0041g02410 } \\
\end{array}$ & 2.19 & 2.66 & ET & SAM-S & VIT_07s0005g02230 & & -3.02 \\
\hline & ABAX & VIT_03s0063g00380 & 2.03 & 2.63 & & & VIT_14s0060g00480 & -1.50 & -2.58 \\
\hline & PP 2C & VIT_08s0007g06510 & & -1.51 & & $\mathrm{ACO}$ & VIT_05s0049g00310 & 1.56 & 1.98 \\
\hline & & VIT_16s0050g02680 & -1.82 & & & & VIT_07s0005g03060 & & -2.25 \\
\hline & SnRK2 & VIT_07s0197g00080 & 1.87 & 1.73 & & & VIT_08s0007g03040 & 2.14 & 2.54 \\
\hline & & VIT_18s0001g06310 & & 1.64 & & EIN3 & VIT_13s0047g00250 & & 1.66 \\
\hline \multirow[t]{20}{*}{ AUX } & TRY-ATF & VIT_18s0157g00140 & 1.81 & 4.65 & & ERF & VIT_00s0772g00020 & 1.65 & 2.00 \\
\hline & TIR & VIT_05s0020g04830 & 2.36 & 3.12 & & & VIT_01s0011g03070 & & 2.24 \\
\hline & $\mathrm{ABP}$ & VIT_07s0005g05910 & & 1.71 & & & VIT_01s0150g00120 & 1.56 & 1.87 \\
\hline & & VIT_07s0005g05930 & & 1.75 & & & VIT_02s0234g00130 & & 2.02 \\
\hline & & VIT_09s0002g01320 & & 4.42 & & & VIT_04s0008g06000 & & -2.02 \\
\hline & & VIT_18s0086g00590 & & 2.93 & & & VIT_04s0023g00970 & & -1.83 \\
\hline & Aux/IAA & VIT_04s0008g00220 & -2.03 & -2.70 & & & VIT_05s0049g00510 & & 1.79 \\
\hline & & VIT_07s0141g00270 & & -1.64 & & & VIT_05s0077g01860 & & 1.68 \\
\hline & & VIT_07s0141g00290 & -2.21 & -3.33 & & & VIT_07s0031g01980 & & 2.65 \\
\hline & Aux-IP & VIT_03s0038g00930 & -1.77 & -2.94 & & & VIT_07s0141g00690 & -1.60 & \\
\hline & & VIT_03s0038g00940 & & -2.71 & & & VIT_08s0040g03180 & & -2.60 \\
\hline & & VIT_04s0023g00560 & 3.45 & & & & VIT_09s0018g01650 & & -2.46 \\
\hline & & VIT_18s0001g13360 & & -1.72 & & & VIT_12s0059g00280 & & 1.80 \\
\hline & & VIT_18s0072g00660 & -1.57 & -2.32 & & & VIT_13s0019g03550 & & 1.55 \\
\hline & IAA-IP & VIT_07s0005g00660 & & 1.89 & & & VIT_14s0006g02290 & -2.32 & -2.90 \\
\hline & & VIT_18s0001g13980 & & -1.92 & & & VIT_15s0021g01630 & & 2.63 \\
\hline & ARF & VIT_06s0004g03130 & & -2.52 & & & VIT_15s0046g00310 & -2.12 & -2.50 \\
\hline & GH3.9 & VIT_07s0005g00090 & 1.56 & & & & VIT_15s0046g01390 & & 1.77 \\
\hline & $\mathrm{AEC}$ & VIT_11s0052g00440 & & -1.92 & & & VIT_16s0013g00950 & & 1.64 \\
\hline & & VIT_17s0000g02420 & & -1.67 & & & VIT_16s0013g01000 & 1.63 & \\
\hline \multirow[t]{3}{*}{ BR } & BSL & VIT_00s1197g00010 & 2.03 & & & & VIT_16s0013g01030 & & 2.09 \\
\hline & & VIT_00s1427g00010 & 2.30 & & & & VIT_16s0013g01070 & & 2.10 \\
\hline & BRU1 & VIT_05s0062g00250 & 2.04 & 3.82 & & & VIT_16s0013g01120 & & 2.01 \\
\hline \multirow[t]{8}{*}{ CK } & UDP-GTF & VIT_18s0001g05990 & & -2.84 & & & VIT_16s0100g00400 & & 1.51 \\
\hline & zeatin-GTF & VIT_08s0007g08920 & 1.63 & 3.35 & & & VIT_17s0000g04480 & 1.58 & 1.93 \\
\hline & CYT & VIT_18s0001g14030 & & -1.61 & & & VIT_18s0001g10150 & & 1.64 \\
\hline & $\mathrm{CH}$ & VIT_13s0158g00320 & & 1.73 & & & VIT_18s0001g03240 & & 2.04 \\
\hline & HK & VIT_01s0010g03780 & & 2.22 & & & VIT_18s0001g05850 & & 1.71 \\
\hline & & VIT_04s0008g03460 & & 1.96 & & & VIT_18s0001g08610 & & -1.58 \\
\hline & & VIT_12s0057g00690 & 1.69 & 1.53 & JA & JA-MTF & VIT_18s0001g12880 & -1.62 & -3.29 \\
\hline & AHP & VIT_09s0002g03520 & -1.67 & -3.25 & & & VIT_18s0001g12890 & -1.70 & -3.61 \\
\hline \multirow[t]{14}{*}{ GA } & DELLA & VIT_14s0006g00640 & & 1.74 & & & VIT_18s0001g12900 & -1.64 & -1.93 \\
\hline & GID2 & VIT_07s0129g01000 & & -1.82 & & TIFY & VIT_01s0146g00480 & & 2.26 \\
\hline & & VIT_18s0001g09700 & & -1.69 & SA & SA-MTF & VIT_04s0023g02220 & & 2.55 \\
\hline & GA20ox & VIT_03s $0063 \mathrm{~g} 01150$ & -3.21 & -2.12 & & & VIT_04s0023g02230 & -2.40 & -1.92 \\
\hline & & VIT_03s0063g01260 & -2.55 & -1.91 & & & VIT_04s0023g02240 & -2.35 & -1.77 \\
\hline & & VIT_09s0002g05280 & -1.56 & -2.23 & & & VIT_04s0023g02260 & -2.64 & -2.09 \\
\hline & & VIT_15s0046g02550 & & -2.28 & & & VIT_04s0023g02310 & & 2.44 \\
\hline & GA3ox & VIT_04s0008g04920 & & 1.76 & & & VIT_04s0023g03810 & & -2.10 \\
\hline & GA2ox & VIT_05s0077g00520 & & 1.99 & MAPKs & MAPK & VIT_06s0004g03540 & & 2.101 \\
\hline & & VIT_06s0004g06790 & -1.82 & & & & VIT_04s0023g02420 & & 1.512 \\
\hline & & VIT_10s0116g00410 & & -3.39 & & MAPKK & VIT_11s0016g02970 & & -2.239 \\
\hline & & VIT_19s0140g00120 & & 3.05 & & & VIT_05s0020g02910 & & -3.101 \\
\hline & & VIT_19s0177g00030 & & -2.59 & & & & & \\
\hline & GA-R & VIT_14s0108g00740 & & -2.97 & & & & & \\
\hline
\end{tabular}

ABA: abscisic acid; AO: aldehyde oxidase; ABAX: abscisic acid 8'-hydroxylase; PP 2C: protein phosphatase 2C; SnRK2: serine/threonine-protein kinase SnRK2; AUX: auxin; TRY-ATF: tryptophan aminotransferase-related protein; TIR1 : transport inhibitor response 1; ABP: auxin-binding protein; Aux/IAA: Aux/IAA proteins; Aux-IP: other auxin induced proteins; IAA-IP: other IAA induced proteins; GH3.9: putative indole-3-acetic acid-amido synthetase GH3.9; AEC: auxin efflux carrier component; BR: brassinosteroid; BRU1: brassinosteroid-regulated protein BRU1; BSL: serine/threonine-protein phosphatase BSL3-like; CK: cytokinin, UDP-GTF: UDPglycosyltransferase 85A1; ZEA-GTF: zeatin O-glucosyltransferase; CYT: cytokinin riboside 5'-monophosphate phosphoribohydrolase; CYH: cytokinin dehydrogenase; HK: histidine kinase; AHP: histidine-containing phosphotransfer protein; GA: gibberellin; DELLA: DELLA protein GAl1; GID2: F-box protein GID2; GA20ox: gibberellin 20 oxidase; GA3ox: gibberellin 3-beta-dioxygenase; GA2ox: gibberellin 2-beta-dioxygenase; GA-R: gibberellin-regulated protein; ET: ethylene, SAM-S: Sadenosylmethionine synthase; ACO: 1-aminocyclopropane-1-carboxylate oxidase; EIN3: ethylene insensitive 3-like; ERF: ethylene-responsive transcription factor; JA: jasmonic acid; JA-MTF: jasmonate O-methyltransferase; TIFY: TIFY 9 protein; SA: salicylic acid; SA-MTF: salicylate O-methyltransferase; MAPKs: mitogen-activated protein kinase cascade: MAPK: mitogen-activated protein kinase; MAPKK: mitogen-activated protein kinase kinase

Up-regulation is marked as green and down-regulation as red. Data were obtained from 3 independent biological replicates

Cofactors metabolism-related pathways were also significantly altered, of which thiamine metabolism was the most represented pathway, followed by vitamin B6 metabolism, riboflavin metabolism, pantothenate and CoA biosynthesis and nicotinate and nicotinamide metabolism at transcriptome level. Phosphate and methylphosphate metabolite levels increased in shade-treated inflorescences.

Secondary metabolic pathways such as phenylpropanoid, stilbenoid, monoterpenoid, diterpenoids, carotenoids, benzenoids, flavonoids and anthocyanin biosynthesis 
and degradation and cytochrome P450-related pathways were significantly affected during shade in both time points. DEG encoding phenylalanine ammonia-lyases (PALs) (EC 4.3.1.25) which catalyse the first step of phenylpropanoids biosynthetic pathway, and stilbene synthases (EC 2.3.1.95) were predominantly up-regulated. Genes encoding myrcene synthases (EC 4.2.3.20) were upregulated while (3S)-linalool/(E)-nerolidol /(E,E)-geranyl linalool synthases (EC 4.2.3.25) were down-regulated. Flavonoids and diterpenoids biosynthetic pathways were, conversely, repressed. At the metabolomic level, oleanolate from terpenoids metabolism, ferulate from phenylpropanoid metabolism, and both $\alpha$ - and $\gamma$-tocopherols increased, while arbutrin (benzenoid) and salidroside (phenylpropanoid) were reduced in result of shaded inflorescences.

Shade altered the accumulation of non-enzymatic markers of oxidative stress, including increased reduced glutathione (GSH) relative content and decreased ascorbate-related metabolites (Additional file 9). The expression of genes encoding enzymatic antioxidants comprising superoxide dismutase, ascorbate oxidase, ascorbate peroxidase, glutathione peroxidase, peroxiredoxin, thioredoxin, glutaredoxin and glutathione Stransferase was also significantly affected (Table 9). In addition, genes encoding laccase (EC 1.10.3.3), involved in ascorbate metabolism and lignin biosynthesis, were exclusively down-regulated in shade treatment.

\section{Shade-responsive transcription factors}

A high number of differentially expressed transcription factors induced by shade treatment was identified, predominantly at 7d, including MYB, GATA, MADS-box, HEX, GT-2, WRKY, CCAAT, ZF-HD, HSF, WOX, E2F/ DP, bHLH, MOT2, MEIS1, RF2b and ZFF (Table 10). In particular, genes encoding MYB and GATA families were the most represented and were predominantly down- and up-regulated, respectively. Transcription factors directly involved in hormone signal transduction pathways were represented in Table 8.

\section{Common DEG and metabolites that significantly changed in response to GAC and shade}

In addition to the DEG found to be specific for each treatment, 36 annotated genes were differentially expressed in both abscission inducing treatments, from which 5 DEG changed with a opposite expression pattern, whereas 31 changed followed the same trend (Table 11). The latter ones could be candidate genes involved in shared pathways leading to abscission and were predominantly up-regulated in response to both stimuli. Only five out of these genes, encoding a cooper transporter, subtilisin-like protease, cytochrome P450, a subunit of exocyst complex, and MYB transcription factor, were down-regulated. Genes that showed an opposite change in expression pattern encode a UGT74B1, glucose-6-phosphate translocator, blue $\mathrm{Cu}$-protein and were up-regulated in GAc treatment and downregulated in shade. Additionally, a gene encoding an ethylene-responsive transcription factor was up-regulated in shade while was repressed in samples from the GActreatment.

Among the 13 commonly altered metabolites in response to both thinning strategies, eight showed the same pattern in both imposed treatments, belonging mostly to the amino acids pathway (Table 12).

On other hand, the phospholipid phosphoethanolamine and nicotianamine decreased in GAc treated samples and increased in those from shaded vines, while putrescine, inosine, arbutine and salidorise were increased in GAc- and reduced in shade-treated inflorescences (Tables 5 and 12). Other gene family, vacuolar $\mathrm{H}^{+}$-ATPase, was affected by GAc and shade treatments, although not exactly the same genes were involved (Additional file 6).

\section{Discussion}

\section{What makes a flower to abscise?}

Flower abscission depicted by -OMIC approaches disclosed a complex regulation including adjustments of metabolism, gene expression and physiology. In grapevine, natural flower drop occurs between 6 and 12 days after $100 \%$ cap fall (d) [62] and peaks at $10 \mathrm{~d}$ under our experimental conditions (data not shown). Our data revealed that GAc and shade induced flower abscission by opposite effects on cell metabolism at 5 and $7 \mathrm{~d}$, but converging on some common pathways leading to abscission.

As previously reported, polyamine metabolism pathway have a key role in reproductive organs abscission $[12,15,16,63,64]$. Changed putrescine inflorescence content varied with the imposed treatment, increasing and decreasing in result of GAc- and shade-treatment, respectively. Whereas putrescine catabolism, by conversion on $\mathrm{N}$-acetylputrescine and/or biosynthesis of downstream polyamines spermidine and spermine with the accumulation of 5-methylthioadenosine (MTA), was affected in the same direction in both treatments (Table 12). MTA is produced from S-adenosylmethionine (SAM) through the spermidine and spermine biosynthetic pathway, where it behaves as a powerful inhibitory product [65], suggesting that the regulation of the downstream polyamines biosynthetic step, but not the biosynthesis of its precursor putrescine, is a common signal of abscission. In addition, in inflorescences developing under shaded conditions, the observed up-regulation of a gene encoding SAM decarboxylase (EC 4.1.1.50) and repression of the subsequent step of spermidine biosynthesis, by the downregulation of a putative SPERMIDINE SYNTHASE 2 gene 
Table 9 DEG encoding oxidative stress-related enzymes in shade-treated inflorescences and respective fold-change

\begin{tabular}{|c|c|c|c|c|c|c|c|}
\hline $\begin{array}{c}\text { Gene } \\
\text { family }\end{array}$ & GID & SH5d & SH7d & $\begin{array}{l}\text { Gene } \\
\text { family }\end{array}$ & GID & SH5d & SH7d \\
\hline \multirow[t]{4}{*}{$\mathrm{AO}$} & VIT_00s0253g00040 & & -2.19 & GST & VIT_01s0026g01340 & -2.67 & -4.08 \\
\hline & VIT_06s0009g01320 & & -2.20 & & VIT_01s0026g01340 & -2.67 & -4.08 \\
\hline & VIT_10s0116g01610 & & -2.80 & & VIT_05s0049g01090 & -1.75 & \\
\hline & VIT_18s0001g00470 & & -2.46 & & VIT_05s0049g01120 & -1.74 & \\
\hline APX & VIT_06s0004g03550 & & -1.86 & & VIT_05s0051g00180 & & 2.01 \\
\hline \multirow[t]{9}{*}{ PX } & VIT_02s0012g00540 & & 2.04 & & VIT_05s0051g00240 & & 2.87 \\
\hline & VIT_03s0063g01040 & 2.63 & 2.90 & & VIT_06s0004g03690 & -1.66 & -2.78 \\
\hline & VIT_05s0077g00880 & & -2.47 & & VIT_06s0004g05690 & & 2.32 \\
\hline & VIT_06s0004g07770 & & -2.05 & & VIT_06s0004g05700 & & 2.11 \\
\hline & VIT_07s0129g00360 & & 1.91 & & VIT_07s0005g04880 & & 2.41 \\
\hline & VIT_07s0130g00220 & & -2.87 & & VIT_07s0005g04880 & & 2.41 \\
\hline & VIT_10s0116g01780 & -1.81 & -3.41 & & VIT_07s0005g04890 & & 2.01 \\
\hline & VIT_14s0066g01850 & & -2.70 & & VIT_07s0104g01800 & -2.09 & -3.16 \\
\hline & VIT_19s0085g01040 & -1.69 & & & VIT_07s0104g01800 & -2.09 & -3.16 \\
\hline SOD & VIT_16s0013g00260 & & -2.29 & & VIT_07s0104g01810 & -2.43 & -3.62 \\
\hline \multirow[t]{3}{*}{ PXR } & VIT_05s0020g00600 & & 1.91 & & VIT_07s0104g01810 & -2.43 & -3.62 \\
\hline & VIT_11s0016g00560 & -2.00 & -3.69 & & VIT_07s0104g01820 & & -2.61 \\
\hline & VIT_11s0016g03630 & & -2.47 & & VIT_07s0104g01820 & & -2.61 \\
\hline \multirow[t]{10}{*}{ TR } & VIT_00s0532g00030 & & 1.98 & & VIT_07s0104g01830 & & -1.77 \\
\hline & VIT_03s0038g04160 & & 2.18 & & VIT_08s0007g01420 & & -1.96 \\
\hline & VIT_04s0008g02900 & & 2.00 & & VIT_08s0007g01420 & & -1.96 \\
\hline & VIT_04s0023g02700 & & -1.58 & & VIT_12s0028g00920 & & 3.56 \\
\hline & VIT_08s0007g07620 & & 1.52 & & VIT_13s0067g03470 & & -1.80 \\
\hline & VIT_17s0000g06370 & & 1.66 & & VIT_16s0039g01070 & & 2.53 \\
\hline & VIT_18s0001g00820 & & -2.08 & & VIT_18s0001g00690 & & -2.03 \\
\hline & VIT_18s0001g13240 & -2.05 & -2.08 & & VIT_18s0001g00690 & & -2.03 \\
\hline & VIT_18s0001g15310 & -2.93 & -3.94 & & VIT_19s0015g02590 & & 2.53 \\
\hline & VIT_19s0014g05090 & 3.31 & 3.14 & & VIT_19s0015g02690 & & 2.44 \\
\hline \multirow[t]{11}{*}{ GR } & VIT_02s0025g01710 & -4.39 & -2.30 & & VIT_19s0015g02730 & & 1.58 \\
\hline & VIT_02s0025g02700 & & -3.42 & & VIT_19s0015g02730 & & 1.58 \\
\hline & VIT_04s0008g01120 & -1.76 & -1.81 & & VIT_19s0015g02880 & & 2.60 \\
\hline & VIT_05s0020g01750 & 3.18 & & & VIT_19s0015g02890 & & 1.54 \\
\hline & VIT_05s0020g01760 & 2.63 & 3.18 & & VIT_19s0015g02890 & & 1.54 \\
\hline & VIT_07s0104g01390 & -2.15 & -3.33 & & VIT_19s0027g00460 & & 2.00 \\
\hline & VIT_07s0104g01400 & 2.37 & & & VIT_19s0027g00460 & & 2.00 \\
\hline & VIT_08s0007g03220 & & -3.53 & & VIT_19s0093g00160 & & 2.57 \\
\hline & VIT_11s0052g00500 & & -1.64 & & VIT_19s0093g00220 & & 1.98 \\
\hline & VIT_13s0067g01650 & & 1.68 & & VIT_19s0093g00220 & & 1.98 \\
\hline & VIT_13s0073g00520 & & -3.50 & & VIT_19s0093g00260 & & 2.18 \\
\hline GPX & VIT_05s0102g00120 & & -3.97 & & VIT_19s0093g00320 & 2.05 & 1.86 \\
\hline
\end{tabular}

AO: ascorbate oxidase; APX: ascorbate peroxidase; PX: peroxidase; SOD: superoxide dismutase; PXR: peroxiredoxin; TR: thioredoxin; GR: glutaredoxin; GPX: glutathione peroxidase; GST: glutathione S-transferase

Up-regulation is marked green and down-regulation is marked red

(VIT_17s0000g08030) indicates that this step of polyamines metabolism was also regulated at transcriptome level (Additional file 6). This is in accordance with observations of abscission inhibition by application of exogenous spermidine, but not of putrescine, prior to flowering [15]. MTA is also produced via ethylene biosynthetic pathway [66] which was significantly affected only by shade treatment (Table 8), while the expression of ERF RAP2-3 was induced by shade and repressed by GAc treatment, thus suggesting that the ethylene signal transduction pathway was differentially regulated according to the treatment (Table 11).

Two common events were the up-regulation of both genes involved in RNA metabolism, such as those encoding RNA polymerases and ribosomal proteins, and energy production related genes, such as NADH dehydrogenases, cytochrome $c$ and ATPase (Table 11), suggesting an increased demand for energy. NADH:ubiquinone oxidoreductase from $\mathrm{NADH}$ dehydrogenase family and cytochrome $c$ are members of the respiratory chain, acting to generate a proton gradient which is thereafter used for ATP synthesis through $\mathrm{H}^{+}$-transporting ATPase. The up- regulation of chloroplastic $\mathrm{NADH}$ dehydrogenases suggested that chlororespiration, which is associated with ROS alleviation around photosystems [67], is also induced as response to both treatments.

In addition to genes encoding serine/threonine protein kinases and calmodulin protein, which are components of signal transduction pathways, a gene encoding a subtilisin-like protease, described to be involved in protein turnover, generation and processing of peptide signals and programmed cell death [68-70], was commonly affected by abscission-inducing stimulus (Table 11). The higher transcript accumulation of a gene encoding a specific antioxidant 1-cys peroxiredoxin enzyme (EC 1.11.1.15), which is prone to be reduced by ascorbic acid or glutathione, was additionally found to be common after both abscising inducing treatments. This observation agrees with previous works that described the multiple ROS roles in abscission including signaling, ROS-sugar-hormone cross talk and induction of the expression of CW-degrading enzymes [11, 14]. Other changes on enzymatic and nonenzymatic oxygen stress remediation mechanisms were found to be specific from each abscission-triggering 
Table 10 DEG encoding transcription factors in shade-treated inflorescences and respective fold-change

\begin{tabular}{|c|c|c|c|c|c|c|c|}
\hline & GID & SH5d & SH7d & & GID & SH5d & SH7d \\
\hline \multirow[t]{2}{*}{ bHLH } & VIT_14s0128g00110 & -1.86 & -3.82 & MOT2 & VIT_13s0019g02510 & -1.80 & -2.42 \\
\hline & VIT_18s0001g06650 & & -1.86 & MEIS1 & VIT_06s0009g00410 & & 1.59 \\
\hline \multirow[t]{5}{*}{ СCAAT } & VIT_00s0956g00020 & & -2.12 & MYB & VIT_00s0341g00050 & & -2.10 \\
\hline & VIT_01s0010g03550 & & -4.39 & & VIT_00s1241g00010 & -1.85 & \\
\hline & VIT_06s0080g00460 & & -2.19 & & VIT_02s0025g02210 & -2.26 & -3.75 \\
\hline & VIT_09s0002g01590 & & 1.71 & & VIT_02s0025g02220 & & -2.72 \\
\hline & VIT_11s0016g01480 & & -1.85 & & VIT_04s0008g01800 & -1.69 & \\
\hline \multirow[t]{3}{*}{ E2F/DP } & VIT_08s0007g00170 & & -1.94 & & VIT_04s0008g01810 & -1.61 & -3.81 \\
\hline & VIT_17s0000g07630 & & -2.62 & & VIT_04s0008g01820 & -1.82 & \\
\hline & VIT_18s0001g14110 & & -1.76 & & VIT_04s0008g01830 & & -1.90 \\
\hline \multirow{22}{*}{$\begin{array}{l}\text { GATA- } \\
4 / 5 / 6\end{array}$} & VIT_01s0011g03520 & 2.09 & 2.96 & & VIT_04s0008g03780 & & -2.10 \\
\hline & VIT_01s0011g04240 & 1.56 & & & VIT_05s0020g01100 & -1.74 & \\
\hline & VIT_01s0150g00410 & & 1.81 & & VIT_05s0049g01020 & & 2.13 \\
\hline & VIT_02s0033g00300 & -1.53 & -2.76 & & VIT_05s0049g02260 & 2.26 & \\
\hline & VIT_03s0038g00340 & & -2.44 & & VIT_05s0077g00500 & & 2.04 \\
\hline & VIT_04s0023g01840 & & -2.19 & & VIT_06s0004g04140 & 2.20 & 1.96 \\
\hline & VIT_08s0007g06310 & 1.65 & 1.59 & & VIT_07s0005g01210 & & -5.21 \\
\hline & VIT_09s0002g03750 & & -2.60 & & VIT_07s0005g01950 & & 2.16 \\
\hline & VIT_09s0054g00530 & -1.80 & & & VIT_08s0007g00410 & & -1.69 \\
\hline & VIT_09s0054g01620 & & 1.91 & & VIT_08s0007g04830 & & 2.90 \\
\hline & VIT_11s0016g02210 & 1.92 & & & VIT_09s0002g01380 & & -1.97 \\
\hline & VIT_11s0103g00760 & & -2.24 & & VIT_09s0002g01670 & -2.18 & -3.21 \\
\hline & VIT_11s0206g00060 & 1.50 & & & VIT_10s0116g01760 & & 1.52 \\
\hline & VIT_12s0028g00980 & & 1.85 & & VIT_11s0016g01300 & -1.91 & -2.50 \\
\hline & VIT_12s0134g00400 & -1.94 & -2.12 & & VIT_11s0016g01320 & & -6.51 \\
\hline & VIT_13s0067g03390 & & 1.70 & & VIT_11s0016g03750 & & -2.02 \\
\hline & VIT_15s0048g02540 & & 1.83 & & VIT_12s0134g00490 & & -2.35 \\
\hline & VIT_16s0098g00360 & -2.03 & -2.50 & & VIT_13s0064g00570 & & 1.75 \\
\hline & VIT_16s0098g00900 & & 1.59 & & VIT_13s0067g01630 & 1.74 & 2.22 \\
\hline & VIT_17s0000g06570 & & 1.55 & & VIT_14s0006g01280 & -2.03 & \\
\hline & VIT_18s0001g13520 & & 1.54 & & VIT_14s0006g01290 & -2.50 & \\
\hline & VIT_19s0014g05120 & 1.62 & & & VIT_14s0006g01340 & -2.40 & \\
\hline \multirow[t]{7}{*}{ GT-2 } & VIT_00s0558g00020 & & 1.75 & & VIT_14s0006g01620 & -2.73 & -4.46 \\
\hline & VIT_02s0025g03220 & & 1.87 & & VIT_14s0066g01220 & & -2.09 \\
\hline & VIT_04s0008g01850 & & -2.03 & & VIT_14s0083g00120 & & -1.88 \\
\hline & VIT_08s0007g04180 & & -3.95 & & VIT_14s0108g01010 & -1.63 & -2.47 \\
\hline & VIT_08s0058g00200 & & 1.62 & & VIT_15s0046g00170 & -2.65 & -3.42 \\
\hline & VIT_13s0084g00800 & & -2.26 & & VIT_17s0000g02660 & -2.53 & -3.53 \\
\hline & VIT_17s0000g10420 & & -1.72 & & VIT_17s0000g03560 & & 1.87 \\
\hline \multirow[t]{4}{*}{ HSF } & VIT_04s0008g01110 & & -2.10 & & VIT_18s0117g00200 & -1.84 & -4.32 \\
\hline & VIT_06s0009g02730 & & -2.25 & & VIT_18s0117g00210 & -1.96 & -2.47 \\
\hline & VIT__12s002 & & 2.03 & RF2b & VIT_06s0004g08070 & & -1.54 \\
\hline & VIT_18s0001g10380 & & -3.12 & wOX & VIT_01 s001 lg05020 & & -1.92 \\
\hline \multirow[t]{7}{*}{ HEX } & VIT_01s0026g01950 & & -1.80 & & VIT_17s0000g02460 & & -2.17 \\
\hline & VIT_04s0023g01330 & & 1.91 & & VIT_18s0001g10160 & -1.55 & -3.72 \\
\hline & VIT_08s0007g04200 & & -4.21 & WRKY & VIT_01s0010g03930 & & 2.70 \\
\hline & VIT_10s0003g00380 & & 1.93 & & VIT_06s0004g07500 & & 2.59 \\
\hline & VIT_13s0156g00260 & & 1.60 & & VIT_07s0005g01710 & & 2.29 \\
\hline & VIT_14s0066g01440 & -1.56 & & & VIT_08s0058g00690 & & 2.64 \\
\hline & VIT_18s0001g06430 & & -2.65 & & VIT_09s0018g00240 & & 3.26 \\
\hline \multirow{7}{*}{$\begin{array}{l}\text { MADS- } \\
\text { box }\end{array}$} & VIT_00s0211g00110 & -2.40 & -4.92 & & VIT_12s0059g00880 & & 1.81 \\
\hline & VIT_00s0211g00180 & 1.78 & & ZF-HD & VIT_12s0035g00650 & 1.86 & 2.55 \\
\hline & VIT_14s0068g01800 & & 2.04 & & VIT_12s0035g01880 & & -1.76 \\
\hline & VIT_14s0083g01030 & & -1.96 & & VIT_17s0000g00810 & & -2.10 \\
\hline & VIT_16s0022g02380 & & 1.84 & & VIT_18s0001g12580 & & 2.10 \\
\hline & VIT_17s0000g04990 & & 1.86 & ZFF & VIT_06s0004g03180 & & 2.06 \\
\hline & VIT_17s0000g06340 & & 1.72 & & & & \\
\hline
\end{tabular}

Up-regulation is marked by green and down-regulation by red

stimulus. In particular, the accumulation of the antioxidants arbutin, salidroside, and the expression of genes encoding a laccase 4 and other blue $\mathrm{Cu}$-protein were contrasting between the two treatments, indicating different ROS detoxification instruments triggered by GAc or by shade treated inflorescences (Tables 11 and 12).

Regarding amino acid metabolism, the observed induction of lysine and isoleucine biosynthetic pathways revealed that both treatments are abiotic stress-impacted. Lysine is a precursor for glutamate, an important signaling amino acid that regulates plant growth and plant- environment responses [71]. On the other hand, isoleucine is accumulated as a compatible osmolyte, playing a role in plant stress tolerance [72]. In lipid-related pathways, changes in glycerolipids and phospholipids metabolism indicated alterations on cell membrane stability and signaling lipids content [73, 74], as candidates to common markers of abscission.

The common event of increased transcription of genes encoding glycogenin and RuBisCO enzymes, suggests that, in what concerns carbohydrate metabolism, conversion of glucose to the energy storage polymer glycogen 
Table 11 List of DEG simultaneous affected by GAc and shade treatments

\begin{tabular}{|c|c|c|c|c|c|c|c|c|}
\hline Gene ID & GAc5d & GAc7d & SH5d & SH7d & Annotation & UniprotKB & $\mathrm{NCBI}$ & Functional category \\
\hline VIT_14s0066g01960 & 1.71 & 1.77 & & 3.51 & $\begin{array}{l}\text { metalloendoproteinase 1, } \\
\text { predicted }\end{array}$ & F6HV36 & & $\begin{array}{l}\text { Amino acid transport } \\
\text { and metabolism }\end{array}$ \\
\hline VIT_14s0060g00740 & & 1.81 & & 1.80 & $\begin{array}{l}\text { glycosyl transferase, family } \\
8 \text { - glycogenin, predicted }\end{array}$ & D7UA70 & & \multirow[t]{4}{*}{$\begin{array}{l}\text { Carbohydrate transport } \\
\text { and metabolism }\end{array}$} \\
\hline VIT_07s0129g00790 & & 2.02 & 1.77 & & $\begin{array}{l}\text { ribulose-1,5-bisphosphate } \\
\text { carboxylase /oxygenase subunit }\end{array}$ & F6HSX2 & & \\
\hline VIT_01s0026g00630 & & 1.65 & -1.99 & -2.71 & $\begin{array}{l}\text { UDP-glycosyltransferase 74B1, } \\
\text { predicted }\end{array}$ & F6HPK7 & XM_002267629.2 & \\
\hline VIT_06s0004g02710 & & 1.60 & -2.64 & -3.46 & $\begin{array}{l}\text { glucose-6-phosphate/ } \\
\text { phosphate antiporter, } \\
\text { predicted }\end{array}$ & D7SKZ8 & XM_002285193.2 & \\
\hline VIT_15s0046g01600 & & 2.11 & & 2.77 & acidic endochitinase, predicted & F61685 & XM_002279522.2 & $\begin{array}{l}\text { Cell wall/membrane/ } \\
\text { envelope biogenesis }\end{array}$ \\
\hline VIT_18s0001g06580 & & 1.60 & -3.04 & -3.11 & $\begin{array}{l}\text { blue copper protein-like, } \\
\text { predicted }\end{array}$ & F6HOY2 & XM_002285700.3 & $\begin{array}{l}\text { Coenzyme transport } \\
\text { and metabolism }\end{array}$ \\
\hline VIT_00s0733g00010 & & 1.83 & 2.60 & & $\begin{array}{l}\text { ATPase subunit } 1 \\
\text { (mitochondrion) }\end{array}$ & $\mathrm{F} 612 \mathrm{~F} 8$ & & \multirow[t]{11}{*}{$\begin{array}{l}\text { Energy production and } \\
\text { conversion }\end{array}$} \\
\hline VIT_00s0332g00170 & & 1.78 & 2.04 & & $\begin{array}{l}\text { NADH:ubiquinone } \\
\text { oxidoreductase, NDUFS2/49 k } \\
\text { subunit (mitochondrion), } \\
\text { predicted }\end{array}$ & F6HSD9 & & \\
\hline VIT_08s0056g01060 & & 2.25 & 2.29 & & $\begin{array}{l}\text { NADH dehydrogenase subunit } \\
1 \text { (chloroplast) }\end{array}$ & F6HMW4 & & \\
\hline VIT_14s0108g01640 & 1.64 & 2.27 & 2.56 & & $\begin{array}{l}\text { NADH-plastoquinone } \\
\text { oxidoreductase subunit } 2 \\
\text { (chloroplast) }\end{array}$ & F6H5N4 & & \\
\hline VIT_13s0067g03310 & & 2.15 & 1.67 & & ATPase subunit, predicted & F6HC65 & & \\
\hline VIT_01s0011g04110 & & 1.75 & 2.22 & 2.39 & $\begin{array}{l}\text { NADH dehydrogenase subunits } \\
2,5 \text {, predicted }\end{array}$ & F6HEV2 & & \\
\hline VIT_00s0246g00050 & & 1.93 & 2.32 & 2.17 & $\begin{array}{l}\text { NADH dehydrogenase subunits } \\
2,5 \text {, predicted }\end{array}$ & D7TKT1 & & \\
\hline VIT_00s0332g00140 & & 1.61 & 1.70 & & $\begin{array}{l}\text { NADH:ubiquinone } \\
\text { oxidoreductase, NDUFS2/49 } \\
\text { kDa subunit, predicted }\end{array}$ & D7TSH3 & & \\
\hline VIT_00s0246g00070 & & 1.96 & & 2.22 & $\begin{array}{l}\text { NADH:ubiquinone } \\
\text { oxidoreductase, NDUFS2/49 } \\
\text { kDa subunit, predicted }\end{array}$ & F6HMJ6 & & \\
\hline VIT_10s0116g00060 & & 2.04 & 2.18 & & $\begin{array}{l}\text { NADH:ubiquinone } \\
\text { oxidoreductase, NDUFS2/49 } \\
\text { kDa subunit, predicted }\end{array}$ & E0CVJ6 & & \\
\hline VIT_10s0092g00770 & 1.68 & 2.27 & 2.79 & & $\begin{array}{l}\text { cytochrome c biogenesis } \\
\text { C (mitochondrion) }\end{array}$ & F6I3KO & XM_010657573.1 & \\
\hline VIT_03s0110g00360 & & -2.04 & & -1.88 & $\begin{array}{l}\text { copper transporter } 6 \text {-like, } \\
\text { predicted }\end{array}$ & A5AQX0 & XM_003631673.2 & $\begin{array}{l}\text { Inorganic ion transport } \\
\text { and metabolism }\end{array}$ \\
\hline VIT_18s0072g00740 & & -1.55 & -1.68 & -1.75 & $\begin{array}{l}\text { sec5 subunit of exocyst } \\
\text { complex, predicted }\end{array}$ & F6GY22 & & $\begin{array}{l}\text { Intracellular traff., } \\
\text { secretion, vesicular trans. }\end{array}$ \\
\hline VIT_07s0031g00570 & & 1.66 & & 3.41 & lipase, predicted & D7SVX1 & & $\begin{array}{l}\text { Lipid transport and } \\
\text { metabolism }\end{array}$ \\
\hline VIT_18s0001g10330 & & -1.60 & & -4.02 & subtilisin-like protease & $\mathrm{F} 6 \mathrm{H} 1 \mathrm{C} 2$ & & $\begin{array}{l}\text { Posttranslational mod., } \\
\text { protein turn., chap. }\end{array}$ \\
\hline VIT_00s0332g00010 & & 1.81 & 2.13 & & mitochondrial mRNA maturase & F6HSC8 & & \multirow{3}{*}{$\begin{array}{l}\text { RNA processing and } \\
\text { modification }\end{array}$} \\
\hline VIT_00s0332g00030 & 1.57 & 1.83 & 2.23 & & $\begin{array}{l}\text { mitochondrial mRNA maturase, } \\
\text { predicted }\end{array}$ & F6HSD0 & & \\
\hline VIT_08s0007g01910 & & 1.90 & -3.05 & -4.22 & laccase-4, predicted & D7THA7 & XM_002278602.3 & \\
\hline
\end{tabular}


Table 11 List of DEG simultaneous affected by GAc and shade treatments (Continued)

\begin{tabular}{|c|c|c|c|c|c|c|c|c|}
\hline VIT_17s0000g01490 & & -1.81 & -1.88 & -2.05 & $\begin{array}{l}\text { cytochrome P450 94A1-like, } \\
\text { predicted }\end{array}$ & F6GST7 & XM_002279945.2 & $\begin{array}{l}\text { Secondary metabolites } \\
\text { biosynthesis, transport } \\
\text { and catabolism }\end{array}$ \\
\hline VIT_02s0012g00820 & & 2.60 & 2.08 & & $\begin{array}{l}\text { serine/threonine protein kinase, } \\
\text { predicted }\end{array}$ & F6HTCO & & $\begin{array}{l}\text { Signal transduction } \\
\text { mechanisms }\end{array}$ \\
\hline VIT_02s0012g00720 & & 2.65 & 2.14 & & $\begin{array}{l}\text { serine/threonine protein kinase, } \\
\text { predicted }\end{array}$ & D7TTF6 & & \\
\hline VIT_12s0028g02570 & & 1.80 & 2.60 & 3.46 & $\begin{array}{l}\text { calmodulin and related proteins } \\
\text { (EF-Hand superfamily), predicted }\end{array}$ & E0CTM8 & XM_002279084.2 & \\
\hline VIT_08s0056g00900 & 1.76 & 1.87 & 2.03 & 2.12 & $\begin{array}{l}\text { RNA polymerase II, second } \\
\text { largest subunit, predicted }\end{array}$ & F6HMV9 & & Transcripton \\
\hline VIT_14s0108g01010 & -1.50 & -1.90 & -1.63 & -2.47 & $\begin{array}{l}\text { transcription factor, Myb } \\
\text { superfamily, predicted }\end{array}$ & F6H5U7 & & \\
\hline VIT_09s0002g04540 & 1.81 & 2.02 & 1.96 & & $\begin{array}{l}\text { DNA-directed RNA polymerase } \\
\text { subunit beta }\end{array}$ & D7U0KO & & \\
\hline VIT_15s0024g01960 & & 2.39 & 2.28 & & $\begin{array}{l}\text { RNA polymerase III, large } \\
\text { subunit, predicted }\end{array}$ & D7UBC3 & & \\
\hline VIT_05s0077g01860 & & -1.56 & & 1.68 & $\begin{array}{l}\text { ethylene-responsive } \\
\text { transcription factor RAP2-3, } \\
\text { predicted }\end{array}$ & D7SYA3 & XM_002272390.2 & \\
\hline VIT_00s0173g00170 & & 2.10 & 1.74 & 2.33 & $\begin{array}{l}30 \text { ribosomal protein } \mathrm{S7}, \\
\text { chloroplastic, predicted }\end{array}$ & F6HD03 & & Translation \\
\hline VIT_10s0092g00790 & & 2.04 & 2.42 & & $\begin{array}{l}\text { ribosomal protein } \mathrm{S} 19 \text {, } \\
\text { mitochondrial-like, predicted }\end{array}$ & D7U8H6 & & \\
\hline VIT_02s0033g00990 & & 2.55 & 2.71 & & $\begin{array}{l}\text { ribosomal protein S7 } \\
\text { (chloroplast) }\end{array}$ & F61086 & & \\
\hline VIT_16s0039g00380 & & 1.77 & 1.85 & & $\begin{array}{l}\text { mitochondrial/chloroplast } \\
\text { ribosomal protein S14/S29, } \\
\text { predicted }\end{array}$ & F6GZ45 & & \\
\hline VIT_12s0028g00970 & & 2.20 & 1.80 & 2.33 & ribosomal protein $\mathrm{S7}$, predicted & F6HRD9 & & \\
\hline VIT_09s0070g00900 & & 1.87 & 1.92 & 2.37 & ribosomal protein $\mathrm{S7}$, predicted & D7U8C4 & & \\
\hline VIT_09s0070g00920 & & 1.79 & 1.84 & 2.41 & ribosomal protein S7, predicted & D7U8C4 & & \\
\hline VIT_13s0047g00220 & & 2.12 & 2.60 & & $\begin{array}{l}\text { mitochondrial/chloroplast } \\
\text { ribosomal protein S19, predicted }\end{array}$ & D7TF26 & & \\
\hline VIT_02s0033g00980 & & 2.79 & 2.88 & & ribosomal protein $\mathrm{S7}$, predicted & F61085 & & \\
\hline VIT_00s0396g00050 & & 1.81 & 2.67 & & $\begin{array}{l}\text { ribosomal protein } \mathrm{S} 4 \\
\text { (mitochondrion) }\end{array}$ & F6HRU1 & XM_010648649.1 & \\
\hline VIT_05s0020g00600 & & 1.66 & & 1.91 & 1-cys peroxiredoxin & D7T674 & NM_001281268.1 & Other \\
\hline
\end{tabular}

Gene code identification, fold-change, annotation, UniProtKB accession number and KOG functional category are reported. Data were obtained from 3 independent biological replicates. Bold letters indicate the metabolites showing opposite trend in both treatments

and $\mathrm{CO}_{2} / \mathrm{O}_{2}$ fixation were affected in both samples (Table 11). At the metabolite level, the accumulation of fumarate (Table 12) was also reported to be associated to flower shedding in response to the same treatments, under greenhouse conditions [12]. Among the multiple functions of fumarate, are the involvement in $\mathrm{pH}$ regulation, stomatal movement and signaling and as a respiratory substrate during carbon starvation $[75,76]$. Expression of genes encoding vacuolar $\mathrm{H}^{+}$-ATPase genes involved in $\mathrm{pH}$ regulation was affected by both treatments, although exclusive up-regulation was only found in GAc treated inflorescences (Additional file 6). This overexpression agrees with the recent findings of cytosolic alkalization as part of abscission pathways and occurring concomitantly with the execution of organ abscission [77].

Pathogenesis-related genes, as the ACIDIC ENDOCHITI$N A S E$ up-regulation in inflorescences submitted to both treatments (Table 11), are reportedly expressed at the site where organs will be shed during abscission [78, 79], and proposed to act in establishing a defense system at the plant's side. 
Table 12 List of metabolites simultaneous affected by GAc and shade treatments

\begin{tabular}{|c|c|c|c|c|c|c|c|}
\hline Metabolite & GAC5d & GAc7d & SH5d & SH7d & KEGG & Pathway & Super pathway \\
\hline 2-aminoadipate & 0.52 & & & 1.09 & C00956 & Aspartate family & Amino acid and peptide \\
\hline $\mathrm{N}$-acetylputrescine & -0.25 & & -0.92 & -1.39 & $\mathrm{C} 02714$ & Glutamate family & \\
\hline Homostachydrine & 0.71 & & 1.39 & 0.84 & C08283 & & \\
\hline Isoleucine & 0.49 & & 2.26 & 1.83 & C00407 & Branched Chain Amino Acids & \\
\hline 5-methylthioadenosine (MTA) & 1.17 & & 1.42 & & C00170 & Amine & \\
\hline Fumarate & 0.57 & & & 0.46 & C00122 & TCA cycle & Carbohydrate \\
\hline Phosphoethanolamine & -0.39 & & & 1.52 & C00346 & Phospholipids & Lipid \\
\hline 2-linoleoylglycerophosphoinositol & 0.91 & & 0.98 & & & Phospholipids & \\
\hline Nicotianamine & -1.04 & & 1.49 & 1.70 & C05324 & Nicotinamide & Cofactor \\
\hline Inosine & 0.80 & & & -0.55 & C00294 & Purine & Nucleotide \\
\hline Pseudouridine & 0.85 & & & 0.82 & C02067 & Pyrimidine & \\
\hline Arbutine & 0.51 & & -0.72 & & C06186 & Benzenoids & Secondary metabolism \\
\hline Salidroside & 2.02 & & -2.69 & & & Phenylpropanoids & \\
\hline
\end{tabular}

Metabolites, fold-change, KEGG compound number and pathway are reported. Data were obtained from 3 independent biological replicates. Bold letters indicate the metabolites with opposite trend

The GAc abscission inducing mechanism requires energy production and global metabolism stimulation

Although the GAc effect is known to be largely dependent on the microclimate conditions [12], GAc application at bloom was a successful treatment to promote flower abscission (Table 1), and cluster loosening at harvest (Table 2), in 'Thompson Seedless' vines growing in open field conditions.

Considering the higher magnitude of changes on metabolite content observed at $5 \mathrm{~d}$ comparing to $7 \mathrm{~d}$ (Additional file 9), together with the most significant transcriptomic reprogramming noticed only at $7 \mathrm{~d}$ (Fig. 3b), one may hypothesize that the process by which exogenous application of GAc significantly increased flower abscission, could result of non-enzymatic mechanisms. Non-enzymatic reactions are an integral part of metabolism, non-targetable and occurring spontaneously as a consequence of the chemical properties of the metabolites, and including reaction of synthesis, redox, decomposition, replacement and isomerisation analogous to principal enzyme categories [80-82]. For instance, an important contribution of non-enzymatic processes, like oxidation, in the release of seed dormancy related to increased GA levels is well known [80]. Thus, our data suggests that GAc spraying led to different levels of metabolism regulation in the grape inflorescences, resulting in modifications on the levels of amino acids and peptides, nucleotide, carbohydrates, lipids, cofactor and secondary metabolisms, energy production and conversion and signal transduction mechanisms (Additional file 10).

Although the leaf $P_{n}$ values have not been significantly affected by GAc treatment (Table 1), in inflorescences it was observed the up-regulation of two genes encoding photosystem I assembly protein and photosystem II reaction center, as well as three genes encoding RuBisCO, as disclosed by RNA-Seq (Additional file 6). These indicators suggested a global reinforcement of the photosynthetic machinery in inflorescences, what might have been accompanied also by an increase in photorespiration and chlororespiration, since glycolate contents increased accompanied by the up-regulation of seven genes encoding chloroplastic NADH dehydrogenase complex units (Fig. 5). Photorespiration and chlororespiration both involve the oxidation of carbohydrates, the consumption of oxygen and are associated with light energy dissipation $[67,83,84]$. Likewise, respiration seemed be enhanced in GAc treated inflorescences, as revealed by the up-regulation of other 13 genes encoding NADH dehydrogenases, one encoding cytochrome $\mathrm{b}$ and two encoding cytochrome c oxidase from respiratory electron transport chain in mitochondria (Fig. 5 and Additional file 6).

These results concerning the photosynthetic and respiration pathways, together with the up-regulation of DEG assigned to sugar and polysaccharide-related pathways, suggested therefore a stimulation of the energy metabolism on inflorescences. This hypothesis is further supported by the accumulation of the precursor ribose, purine and pyrimidine nucleotides (Additional file 9), which play a central role as energy carriers and subunits of nucleic acids, indicating also a global increased in gene expression. Accordingly, the decreased glucose 6phosphate, fructose-1,6-biphosphate and mannose-6phosphate (Fig. 5) suggests a degradation of these molecules to generate ribulose-5-phosphate, which is a precursor of the nucleotides synthesis. Furthermore, the overall induction of nucleotide and 
carbohydrates metabolism in response to GAc used as abscission inducing treatment in grapevine was previously reported [12].

The increased mannitol content is known be related to stress tolerance due to the osmoprotectant function [85]. Only GAc promoted an accumulation of the ascorbateprecursor galactose [86], in agreement with the increase in dehydroascorbate previously reported in a different genetic background [12].

GAc caused changes in the inflorescences levels of transcripts and metabolites involved in the secondary metabolism at bloom (Fig. 5), similarly to what was previously observed in an earlier phenological stage (pre-bloom) [30]. The quercetin-3-O-glucoside and naringenin accumulation and repression of a gene encoding a hyoscyamine 6-dioxygenase suggests induced flavonoids metabolism. In particular, a gene encoding an UDP-glycosyltransferase 74B1, proposed to be involved in the secondary metabolism and as a defense response by callose deposition into the $\mathrm{CW}$, is also part of IAA biosynthetic pathway. Hence, the up-regulation of this gene suggests that the increment of auxin contents might be needed for the GAc-induced responses [29]. On the other hand, taking into account the downregulation of GIBBERELLIN 3-BETA-DIOXYGENASE 1 and the increased $G_{8}$ content which results from $G_{1}$ inactivation (Fig. 5 and Table 5), a reduction of the endogenous bioactive GA level can be suggested, probably due to a negative-feedback regulation promoted by GAc spraying, as previously observed after GAc treatments in different phenological stages $[29,30]$. An auxin regulation of bioactive GAc levels has been suggested, corroborating this assumption [87].

\section{Shade induced abscission by nutritional stress and global metabolism repression}

'Thompson Seedless' showed to be sensitive to shade imposed at $50 \%$ cap fall and during 14 days, resulting in increased flower drop percentages (Table 1). These observations at bloom stage together with depicted less compact bunches and lower number of berries at harvest (Table 2), suggests that this approach can be exploited as an alternative method for thinning berries. The observed decline of $P_{n}$ to zero will consequently decrease $\mathrm{C}$-resources available to both vegetative and reproductive sinks. This will increase the competition between sinks [88-90], and promote flower abortion [17]. Our results highlighted also the importance of the $\mathrm{P}_{\mathrm{n}}$ during bloom to the developing cluster, despite the carbohydrate reserves [91]. Shading also affected leaf chlorophyll content, total leaf area and shoot growth (Table 1), showing a more pronounced effect in vegetative growth comparing to previously observations under greenhouse conditions [12]. This might have been related to a higher percentage of light intercepted, to the different genetic background and to the field growing conditions, in the present work.

Shade resulted in the down-regulation of a large group of genes involved in photosynthesis, carbohydrates metabolism and transport [18, 92], and reduced carbon and carbon derived metabolites content [12, 15] (Additional file 6 and Additional file 9). The accumulation of arabonate and xylonate were the only exceptions at the metabolite level. These monosaccharides decorate $\mathrm{CW}$ polymers, such as pectins or xyloglucans, and its presence can result from $\mathrm{CW}$ remodeling processes that occur during pedicel AZ formation, protective layer differentiation on the proximal side after organ detachment [93] and alterations on CW structure and growth in adaptation to the imposed abiotic stress [94]. Their accumulation is also in accordance to the differentially expression of pectinesterases (EC 3.1.1.11), polygalacturonases (EC 3.2.1.15), expansins, cellulose synthase (EC 2.4.1.12) and callose synthase (EC 2.4.1.34). Consistently, starch and sucrose metabolism was the most represented affected pathway which is reported to be very sensitive to environment changes, providing the mobilization of stored carbohydrates [95] (Additional file 11). The analysis of the impact of shade on sugar signaling pathway and transport (Table 7) showed that expression of genes from Sucrose Nonfermenting-1 (SNF1) Related protein Kinases1(SnRK1) family was significantly affected, which were identified as central regulators of the transcriptome in response to darkness and multiple types of stress signals triggering extensive transcriptional changes [96]. The predominant up-regulation of trehalose-6-phosphate synthase (EC 2.4.1.15) genes is also involved in SnRK1 signaling [97]. Hexose kinases and invertases (EC 3.2.1.26, 3.2.1.48), involved in sugar signaling [98, 99], showed to be implicated in organ abscission via shading, as previously reported [18]. Sucrose mobilization was also induced during shade, through the up-regulation of a gene encoding the reversible sucrose synthase (EC 2.4.1.13), indicating altered sucrose and sucrose-derived metabolites and sucrose-specific signaling pathway [100].

At transcriptomic and metabolomic levels, shade imposition led to a classic signature of carbon/nitrogen $(\mathrm{C} /$ $\mathrm{N}$ ) imbalance due to carbon deficit, with a stimulation of amino acids metabolism, a repression of energy metabolites and carbon metabolites pathways and increased accumulation of oxidative stress markers [95, 101]. According to the amino acid and peptide biosynthesis, metabolism and transport associated pathways affected by the shade treatment, the increased content of the proteinogenic amino acids may result from amino acid biosynthesis and from enhanced protein turnover to free up amino acid carbon backbones for energy utilization. 
Particularly, the increased aromatic amino acids phenylalanine, tyrosine and tryptophan contents might result from stress- induced protein breakdown, as revealed by the decline of the biosynthetic precursor shikimate levels, simultaneous with the down-regulation of genes encoding enzymes of the shikimate pathway, as shikimate kinase (EC 2.7.1.71) and the bi-functional enzyme 3-dehydroquinate dehydratase/shikimate 5dehydrogenase (EC 4.2.1.10) (Additional file 11). Our results are in accordance with previous studies which demonstrate that abiotic stresses enhance accumulation of beatine, proline and allantoin [95, 102, 103]. Allantoin, which was the mostly increased metabolite in inflorescences developing under shade (Additional file 9), often accumulates as a response to $\mathrm{C} / \mathrm{N}$ imbalances, and results from purine degradation is implicated in nitrogen metabolism and stress tolerance by activation of abscisic acid metabolism [104].

Our data suggests that, under shade imposition, ABA biosynthesis, catabolism and signaling pathways were stimulated (Table 8). The effect of ABA in abscission can be directly related to the activation of ABA-signaling genes and/ or indirectly associated to an ACC increase and to ethylene biosynthesis $[8,11]$. On the other hand, ethylene accumulation can promote ABA catabolism as a consequence of increased ABA 8'-hydroxylase activity [105], which resulted in a reduced net ABA content (Table 5). The decreased ABA content was also observed by [106], as response from the soybean reproductive structures to shading.

Ethylene-auxin balance is recognized as one of the most important regulators of organ abscission determination $[9,48]$. The acquisition of sensitivity to ethylene by the AZ cells has been associated with an altered expression of auxin-regulated genes as a result of auxin depletion [7]. Moreover, [48] showed that auxin regulates the timing of organ abscission and that a functional IAA signaling pathway is required for setting up the event. Our data shows that auxin biosynthesis was induced in shaded-treated inflorescences, and the auxin signaling pathway was active with the up-regulation of genes encoding auxin receptors TIR1 and ABP and downregulation of $A u x / I A A$ and $A R F$ genes in both time points investigated (Table 8 ). On the other hand, the upregulation of a gene encoding an IAA-amido synthetase GH3.9 only at $5 \mathrm{~d}$, as previously observed in shadeinduced lychee abscission [92], indicated that auxin conjugation reducing the free IAA content, can exert an important role in auxin-ethylene balance. Accordingly, auxin transport showed to be repressed by downregulation of $A E C$ genes only at $7 \mathrm{~d}$, as previously reported [18] in response to the fruit abscission induction by naphthaleneacetic acid application.

Ethylene biosynthesis and signal transduction pathways were induced in shade treated inflorescences, involving the accumulation of cyano-alanine and the predominantly upregulation of genes encoding ACC oxidases and EIN3 and differentially regulation of ERF family of transcription factors involved in activation or repression of transcription activity [3, 107] (Additional file 9 and Table 8). In particular key elements of MAPK cascades related to ABA and ethylene signal transduction pathways [108], and known to be involved in floral organ abscission [20] were regulated, as those coding for MAPK3, MAPK4, MAPKK5 and MAPKK6 (Table 8). In addition, GTPase mediated signal transduction, upstream of MAPK cascades [109], was induced during shade treatment (Table 6) and was previously shown to be involved in leaf abscission signaling and ethylene biosynthesis [110] and to regulate the movement of key molecules required for abscission [111]. GAs biosynthetic and signaling pathways were predominantly repressed, accordingly with [112] that demonstrated that fruit abscission is enhanced by low carbohydrates and GAs availability.

The significant impact on CKs activation, perception and degradation caused by light reduction during bloom (Table 8) highlights the role of this hormones class, and is in accordance with the described CKs action as abscission-accelerating signal [11], although following the hypothesis of having ethylene regulation [113]. Also $\mathrm{BR}, \mathrm{SA}$ and JA metabolisms were correlated with the abscission boost caused by shade (Table 8 ). The accumulation of SA content agrees with the down-regulation of genes encoding salicylate-O-methyltransferase and the general up-regulation of genes encoding PALs (Additional file 6) involved in its own biosynthesis [114]. In addition, the accumulation of oxidized lipids confirmed to occur in response to shade, as 13-HODE and 9-HODE, products of elevated oxidative status, have been linked to the JA biosynthetic pathway [115].

Some of the most striking changes observed in shaded inflorescence samples were represented by DEG and accumulation of metabolites associated with oxygen stress remediation (Table 9 and Additional file 9), and amongst them, the intermediates of the glutathione synthesis cycle were the most represented, as previously reported [12]. On the other hand, ascorbate metabolism seemed to be inhibited, as suggested by the down-regulation of genes encoding ascorbate oxidase, ascorbate peroxidase and GDP-l-galactose phosphorylase, concomitantly with decreased levels of metabolites related with ascorbate metabolism. Regarding secondary metabolism, the fact that flavonoids and diterpenoids-related pathways had been predominantly repressed, while phenylpropanoids and stilbenoid-related pathways were predominantly induced (Additional file 6), pinpoints a slowdown of biochemical reactions while promoting the activation of stress responses and defense systems during abscission [116].

Among the transcription factors differentially regulated by shade treatment, members of MADs-box, AP2, 
MYB, WRKY, zinc finger transcription factor families were previously described to participate in abscission regulation $[7,11,18,92,117]$.

\section{Conclusions}

The two imposed treatments induced flower abscission by exerting different effects on grapevine inflorescences metabolism, agreeing with the mechanistic model previously proposed [12]. GAc treatment response suggested a reinforcement of the energetic metabolism simultaneously with induction of nucleotide biosynthesis and carbon metabolism. A global metabolism stimulation of the central flower (king flower), which open before the smaller lateral ones [33], by GAc application, can be hypothesize, promoting the fruit set of these flowers and the developmental inhibition and abscission of the later ones. On the other hand, shade imposition induced carbohydrate metabolism repression, promoting flower drop by the previously described abscission process via nutritional stress $[11,18]$ associated with sugar-, ethylene- and auxin-responsive signaling pathways and other signaling pathways to coordinate abscission. Regulation of polyamines metabolism, activation of ROS scavenging mechanisms, alterations on ethylene signaling pathway and bioactive GA biosynthesis repression were identified as candidate common signatures of abscission. Our data provided a new insight on alternative pathways leading to abscission, which can assist the development and optimization of strategies for abscission control in fruit crop species.

\section{Availability of supporting data}

The data sets supporting the results of this article have been submitted to the Sequence Read Archive at NCBI (http://www.ncbi.nlm.nih.gov/sra) under accession numbers [SRA:SRX964421, SRX964430, SRX966723, SRX966735, SRX966740, SRX966742, SRX966755, SRX1008101, SRX1008174, SRX1008177, SRX1008181, SRX1008185, SRX1008211, SRX1008213, SRX1008214, SRX1008217, SRX1010114, SRX1010115].

\section{Additional files}

Additional file 1: Figure S1. Microclimate conditions recorded during bloom period (twelve days) under shaded and unshaded conditions. Mean values per hour of relative humidity $(\mathrm{RH})$, temperature and photosynthetic photon flux density (PPFD) ( \pm se). (PDF 155 kb)

Additional file 2: Figure S2. Quantitative rtPCR validation of the RNA-seq. NCBI reference, gene identification (GID), fold change quantification by q-rtPCR and RNA seq, primers and amplicon length (bp) for abscission related-genes (A). NCBI reference, primers and amplicon length are also given for references genes (B). Data are means of 3 replicates. The pearson correlation coefficient between qRT-PCR and RNA-Seq obtained fold-changes $(R=0.84)$ is significant at $p$-value $\leq 0.001$ (C). (PDF $176 \mathrm{~kb}$ )

Additional file 3: Table S1. Reference library of chemical standard used for LC/MS/MS analysis. (XLSX $28 \mathrm{~kb}$ )
Additional file 4: Table S2. Parameters for hormone identification in Mass Spectrometry. Cone voltage potential, collision energy (CE) and other performance characteristics. (PDF $67 \mathrm{~kb}$ )

Additional file 5: Figure S3. Pie charts summarizing the results of alignment of each sample against the Vitis vinifera genome. Percentage of reads by sample, mapped uniquely (black), unmapped (grey) or mapped in multiple locations (white). (PDF 54 kb)

Additional file 6: Table S3. List of genes significantly affected by GAC and shade treatments. Gene annotation, functional categories and respective fold-change. The abbreviations GAc5d, GAc7d, SH5d and SH7d mean the $\log 2$ fold-change between gene relative expression obtained in treated and control inflorescences, at 5 and 7 days after $100 \%$ cap fall. (XLSX $777 \mathrm{~kb})$

Additional file 7: Figure S4. Orthogonal Signal Correction Partial Least Squares Discriminant Analysis (O-PLS-DA) of differential expressed genes (A) and differentially accumulated metabolites (B). The genes and metabolites are grouped and the dispersion color was coded by their KOG categories or super-pathway, respectively. Data was In-transformed prior to the analyses. (PDF $140 \mathrm{~kb}$ )

Additional file 8: Figure S5. Pearson correlation plots of RNA-Seq reads. Correlation between individual biological replicates in each time-point (5 and 7d) and treatment (control, GAc and shade) using In-transformed read counts for the DEG as input. All correlation values are significant at $p$-value $\leq 0.001$. (PDF $140 \mathrm{~kb}$ )

Additional file 9: Table S4. Heat map illustrating metabolite changes in response to GAc and shade treatments. The abbreviations GAc5d, GAC7d, SH5d and SH7d mean the log2 fold-change between metabolite relative content obtained in treated and control inflorescences, at 5 and 7 days after $100 \%$ cap fall. Red and green shaded cells indicate $p$-value $\leq$ 0.05 . Light red and light green shaded cells indicate $0.05<p$-values $\leq$ 0.10. (XLSX $70 \mathrm{~kb})$

Additional file 10: Figure S6. Distribution of differentially changed transcripts and metabolites according to functional categories. Each pie chart corresponds to changes occurred as response to GAc and shade treatments at 5 and 7 days after $100 \%$ cap fall (d). (PDF 234 kb)

Additional file 11: Table S5. Enzymatic classification of differentially expressed genes and respective fold-change for each treatment at 5 and 7d. (XLSX $68 \mathrm{~kb})$

Additional file 12: Table S6. List of $G O$ enriched categories in each pair-wise comparison. Enrichment analysis was performed using $R$ bioconductor topGO package with Fisher's exact test and $p$-value $\leq 0.01$. (XLSX $56 \mathrm{~kb}$ )

Additional file 13: Figure S7. Acyclic graphs with the mostly enriched GO categories in differentially expressed genes. The top 5 and top 5-related biological processes, molecular function and cellular component at GAc $7 d$ (A), SH5d (B) and $\mathrm{SH} 7 \mathrm{~d}(\mathrm{C})$ are shown. The color scale presented is related with the $p$-value, where the redder the node, lower the $p$-value. The nodes with black outline are the top 5 enriched categories. (PDF $440 \mathrm{~kb}$ )

\section{Abbreviations}

ABA: Abscisic acid; ACC: 1-aminocyclopropane-1-carboxylate; ARF: Auxin response factor; AZ: Abscission zone; BR: Brassinosteroid; CK: Ccytokinin; CW: Cell wall; DEG: Differentially expressed genes; d: Days after $100 \%$ cap fall; ERF: Ethylene response factor; IAA: Indole-3-acetic acid; FPKM: Fragments per kilobase of exon per Million fragments mapped; GA: Gibberellin; GAc: Gibberellic acid; GA20ox: Gibberellin20 oxidase; GO: Gene Ontology; 9: Stomatal conductance; JA: Jasmonic acid; KEG: Kyoto Encyclopedia of Genes and Genomes; KOG: euKaryotic Orthologous Groups; MAPK: Mitogen activated protein kinase; PA: Polyamine; PAL: Phenylalanine ammonia-lyase; PCD: Programmed cell death; $P_{n}$ : Net photosynthetic rate; PMN: Plant Metabolic Network; RNA-Seq: Ribonucleic acid sequencing; ROS: Reactive oxygen species; RuBisCO: Ribulose-1,5-bisphosphate carboxylase-oxygenase; SA: Salicylic acid; SAM: S-adenosylmethionine; TCA: Tricarboxylic acid. 


\section{Authors' contribution}

LFG, CMO and SD were responsible for the conception and design of the experiments, SD and $\mathrm{VC}$ were responsible for samples acquisition and RNA extraction, SD, VC, JCR, CS and RL were responsible for physiological and metabolic determinations, JF and OSP carried out transcriptomic bioinformatic analysis. All authors drafted and approved the final manuscript. LFG and CMO steered the project.

\section{Acknowledgments}

This work was supported by the Portuguese Fundação para a Ciência e a Tecnologia (FCT) through project "VitiShade: PTDC/AGR-GPL/116923/2010" and the PhD grant SFRH/BD/69076/2010 to SD, and by ProDeR Action4.1: PROdUVA 23921/2/3/4. We acknowledge Dr Danny Alexander and his team at Metabolon Inc. (Durham, USA) for metabolomic analysis, and Dr Martin Heine and his team at LGC Genomics (Berlin, Germany) for transcriptome sequencing.

\section{Author details}

${ }^{1}$ Linking Landscape, Environment, Agriculture and Food (LEAF), Instituto Superior de Agronomia (ISA), Universidade de Lisboa (ULisboa), Lisbon, Portugal. ${ }^{2}$ Instituto de Investigação Científica Tropical, I.P. (IICT), Lisbon, Portugal. ${ }^{3}$ Computational Biology and Population Genomics Group, Centre for Ecology, Evolution and Environmental Changes (cE3c), Faculdade de Ciências, Universidade de Lisboa, Lisbon, Portugal. ${ }^{4}$ Instituto Nacional de Investigação Agrária e Veterinária, I.P. (INIAV), Oeiras, Portugal. ${ }^{5} \mathrm{GeoBioTec}$ Faculdade de Ciências e Tecnolgia (FCT), Universidade Nova de Lisboa (UNL), Caparica, Portugal. ${ }^{6}$ FEM-IASMA, Fondazione Edmund Mach, Istituto Agrario di San Michele all'Adige, San Michele all'Adige, TN, Italy. ${ }^{7}$ Present address: Colégio Food, Farming and Forestry, Universidade de Lisboa (ULisboa), Lisbon, Portugal.

\section{Received: 6 October 2015 Accepted: 21 January 2016}

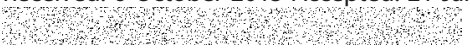

\section{References}

1. Bonghi C, Tonutti P, Ramina A. Biochemical and molecular aspects of fruitlet abscission. Plant Growth Regul. 2000;31:35-42.

2. Sawicki M, Aït Barka E, Clément C, Vaillant-Gaveau N, Jacquard C. Cross-talk between environmental stresses and plant metabolism during reproductive organ abscission. J Exp Bot. 2015;66(7):1707-19.

3. Taylor JE, Whitelaw CA. Signal in abscission. New Phytol. 2001;151:323-39.

4. Patterson SE. Cutting loose. Abscission and dehiscence in Arabidopsis. Plant Physiol. 2001;126(2):494-500.

5. Estornell LH, Agustí J, Merelo P, Talón M, Tadeo FR. Elucidating mechanisms underlying organ abscission. Plant Sci. 2013;199-200:48-60.

6. Else MA, Stankiewicz-Davies AP, Crisp CM, Atkinson CJ. The role of polar auxin transport through pedicels of Prunus avium $\mathrm{L}$. in relation to fruit development and retention. J Exp Bot. 2004;55:2099-109.

7. Meir S, Philosoph-Hadas S, Sundaresan S, Selvaraj KSV, Burd S, Ophir R, et al. Microarray analysis of the abscission-related transcriptome in the tomato flower abscission zone in response to auxin depletion. Plant Physiol. 2010; 154(4):1929-56.

8. Gomez-Cadenas A, Mehouachi J, Tadeo FR, Primo-Millo E, Talon M. Hormonal regulation of fruitlet abscission induced by carbohydrate shortage in citrus. Planta. 2000;210:636-43.

9. Dal Cin V, Danesin M, Boschetti A, Dorigoni A, Ramina A. Ethylene biosynthesis and perception in apple fruitlet abscission (Malus domestica L. Borck). J Exp Bot. 2005;56:2995-3005.

10. Singh AP, Tripathi SK, Nath P, Sane AP. Petal abscission in rose is associated with the differential expression of two ethylene-responsive xyloglucan endotransglucosylase/hydrolase genes, RbXTH1 and RbXTH2. J Exp Bot. 2011;62(14):5091-103.

11. Botton A, Eccher G, Forcato C, Ferrarini A, Begheldo M, Zermiani M, et al. Signaling pathways mediating the induction of apple fruitlet abscission. Plant Physiol. 2011;155(1):185-208.

12. Domingos S, Scafidi P, Cardoso V, Leitao AE, Di Lorenzo R, Oliveira CM, et al. Flower abscission in Vitis vinifera L. triggered by gibberellic acid and shade discloses differences in the underlying metabolic pathways. Front Plant Sci. 2015;6:457.
13. Giulia E, Alessandro B, Mariano D, Andrea B, Benedetto R, Angelo R. Early induction of apple fruitlet abscission is characterized by an increase of both isoprene emission and abscisic acid content. Plant Physiol. 2013;161(4):1952-69.

14. Sakamoto M, Munemura I, Tomita R, Kobayashi K. Reactive oxygen species in leaf abscission signaling. Plant Signal Behav. 2008;3(11):1014-5.

15. Aziz A. Spermidine and related metabolic inhibitors modulate sugar and amino acid levels in Vitis vinifera L: possible relationships with initial fruitlet abscission. J Exp Bot. 2003;54:355-63.

16. Gomez-Jimenez MC, Paredes MA, Gallardo M, Sanchez-Calle IM. Mature fruit abscission is associated with up-regulation of polyamine metabolism in the olive abscission zone. J Plant Physiol. 2010;167(17):1432-41.

17. Lebon G, Wojnarowiez G, Holzapfel B, Fontaine F, Vaillant-Gaveau N, Clément C. Sugars and flowering in the grapevine (Vitis vinifera L.). J Exp Bot. 2008;59:2565-78.

18. Zhu H, Dardick C, Beers E, Callanhan A, Xia R, Yuan R. Transcriptomics of shading-induced and NAA-induced abscission in apple (Malus domestica) reveals a shared pathway involving reduced photosynthesis, alterations in carbohydrate transport and signaling and hormone crosstalk. BMC Plant Biol. 2011;11:138.

19. Ruan Y-L, Patrick JW, Bouzayen M, Osorio S, Fernie AR. Molecular regulation of seed and fruit set. Trends Plant Sci. 2012;17(11):656-65.

20. Cho SK, Larue CT, Chevalier D, Wang H, Jinn T-L, Zhang S, et al. Regulation of floral organ abscission in Arabidopsis thaliana. Proc Natl Acad Sci U S A. 2008;105(40):15629-34.

21. Niederhuth CE, Patharkar OR, Walker JC. Transcriptional profiling of the Arabidopsis abscission mutant hae hsl2 by RNA-Seq. BMC Genomics. 2013; 14:37.

22. Tucker ML, Yang R. IDA-like gene expression in soybean and tomato leaf abscission and requirement for a diffusible stelar abscission signal. AoB Plants. 2012;2012:pls035.

23. Dokoozlian NK, Peacock WL. Gibberellic acid applied at bloom reduces fruit set and improves size of "Crimson Seedless" table grapes. HortScience. 2001; 36(4):706-9.

24. Hed B, Ngug HK, W. TJ. Use of gibberellic acid for management of bunch rot on chardonnay and vignoles grape. Plant Dis. 2011;95(3):269-78.

25. Looney NE, Wood DF. Some cluster thinning and gibberellic acid effects on fruit set, berry size, vine growth and yiels of De Chaunac grapes. Can J Plant Sci. 1977:57:653-9.

26. Reynolds AG, Savigny C. Influence of girdling and gibberellic acid on yield components, fruit composition and vestigial seed formation of "Sovereign Coronation" table grapes. HortScience. 2004;39(3):541-4.

27. Reynolds AG, Roller JN, Forgione A, De Savigny C. Gibberellic acid and basal leaf removal: implications for fruit maturity, vestigial seed development, and sensory attributes of Sovereign Coronation table grapes. Am J Enol Vitic. 2006;57:41-53.

28. Sun T. Gibberellin-GID1-DELLA: a pivotal regulatory module for plant growth and development. Plant Physiol. 2010;154(2):567-70.

29. Chai L, Li Y, Chen S, Perl A, Zhao F, Ma H. RNA sequencing reveals high resolution expression change of major plant hormone pathway genes after young seedless grape berries treated with gibberellin. Plant Sci. 2014;229: 215-24

30. Cheng C, Jiao C, Singer SD, Gao M, Xu X, Zhou Y, et al. Gibberellin-induced changes in the transcriptome of grapevine (Vitis labrusca $\times V$. vinifera) $\mathrm{cV}$. Kyoho flowers. BMC Genomics. 2015;16(1):128.

31. Ferree DC, McArtney SJ, Scurlock DM. Influence of irradiance and period of exposure on fruit set of french-american hybrid grapes. J Am Soc Hortic Sci. 2001;126:283-90.

32. Roubelakis KA, Kliewer WM. Influence of light intensity and growth regulators on fruit-set and ovule fertilization in grape cultivars under low temperature conditions. Am J Enol Vitic. 1976;27:163-7.

33. Vasconcelos MC, Greven M, Winefield CS, Trought MCT, Raw V. The flowering process of Vitis vinifera: a review. Am J Enol Vitic. 2009;60(4):411-34.

34. Perez FJ, Viani C, Retamales J. Bioactive gibberellins in seeded and seedless grapes: identification and changes in content during berry development. Am J Enol Vitic. 2000;51(4):315-8.

35. Lorenz D, Eichhorn K, Bleiholder H, Klose R, Meier U, Weber E. Phänologische Entwicklungsstadien der Rebe (Vitis vinifera L. ssp. vinifera). Codierung und Beschreibung nach der erweiterten BBCH-Skala. Vitic Enol Sci. 1994;49:66-70.

36. Chang S, Puryear J, Cairney J. A simple and efficient method for isolating RNA from pine trees. Plant Mol Biol Report. 1993;11(2):113-6. 
37. Bolger AM, Lohse M, Usadel B. Trimmomatic: a flexible trimmer for Illumina sequence data. Bioinformatics. 2014;30(15):2114-20.

38. Quast C, Pruesse E, Yilmaz P, Gerken J, Schweer T, Yarza P, et al. The SILVA ribosomal RNA gene database project: improved data processing and webbased tools. Nucleic Acids Res. 2013;4:D590-6.

39. Jaillon O, Aury J-M, Noel B, Policriti A, Clepet C, Casagrande A, et al. The grapevine genome sequence suggests ancestral hexaploidization in major angiosperm phyla. Nature. 2007:449(7161):463-7.

40. Kim D, Pertea G, Trapnell C, Pimentel H, Kelley R, Salzberg SL. TopHat2: accurate alignment of transcriptomes in the presence of insertions, deletions and gene fusions. Genome Biol. 2013;14(4):R36.

41. Trapnell C, Williams BA, Pertea G, Mortazavi A, Kwan G, van Baren MJ, et al. Transcript assembly and quantification by RNA-Seq reveals unannotated transcripts and isoform switching during cell differentiation. Nat Biotechnol. 2010;28(5):511-5.

42. Love Ml, Huber W, Anders S. Moderated estimation of fold change and dispersion for RNA-seq data with DESeq2. Genome Biol. 2014;15(12):550.

43. Tatusov RL, Fedorova ND, Jackson JD, Jacobs AR, Kiryutin B, Koonin EV, et al. The COG database: an updated version includes eukaryotes. BMC Bioinformatics. 2003:4:41.

44. Kanehisa M, Araki M, Goto S, Hattori M, Hirakawa M, Itoh M, et al. KEGG for linking genomes to life and the environment. Nucleic Acids Res. 2007;36: D480-4.

45. Zhao $Y$, Tang H, Ye Y. RAPSearch2: a fast and memory-efficient protein similarity search tool for next-generation sequencing data. Bioinformatics. 2012;28(1):125-6.

46. Conesa A, Götz S, García-Gómez JM, Terol J, Talón M, Robles M. Blast2GO: a universal tool for annotation, visualization and analysis in functional genomics research. Bioinformatics. 2005;21(18):3674-6.

47. Alexa A, Rahnenfuhrer J. topGO: enrichment analysis for gene ontology. R Packag version. 2010;2.0

48. Basu MM, González-Carranza ZH, Azam-Ali S, Tang S, Shahid AA, Roberts JA. The manipulation of auxin in the abscission zone cells of Arabidopsis flowers reveals that indoleacetic acid signaling is a prerequisite for organ shedding. Plant Physiol. 2013;162(1):96-106.

49. Rasmussen R. Quantification on the light cycler. In: Meuer S, Wittwer C, Nakagawara K-I, editors. Rapid cycle real-time PCR, methods and applications. Berlin, Heidelberg: Springer Berlin Heidelberg; 2001. p. 21-34.

50. Vandesompele J, Kubista M, Pfaffl M. Reference gene validation software for improved normalization. In: Logan JMJ, Edwards KJ, Saunders NA, editors. Real-time PCR: current technology and applications. Norfolk: Caister Academic; 2009. p. 47-63.

51. Evans A, DeHaven C, Barrett T. Integrated, nontargeted ultrahigh performance liquid chromatography/electrospray ionization tandem mass spectrometry platform for the identification and relative. Anal Chem. 2009; 81(16):6656-67.

52. Evans AM, Mitchell MW, Dai H, DeHaven CD. Categorizing ion -features in liquid chromatography/mass spectrometry metobolomics data. Metabolomics. 2012;2:111.

53. DeHaven CD, Evans AM, Dai H, Lawton KA. Organization of GC/MS and LC/ MS metabolomics data into chemical libraries. J Cheminform. 2010;2.

54. Giacomelli L, Rota-Stabelli O, Masuero D, Acheampong AK, Moretto M, Caputi $L$, et al. Gibberellin metabolism in Vitis vinifera $\mathrm{L}$. during bloom and fruit-set: functional characterization and evolution of grapevine gibberellin oxidases. J Exp Bot. 2013;64(14):4403-19.

55. Rohlf FJ. NTSYS-pc: numerical taxonomy and multivariate analysis system. Setauket, New York, USA: Exeter Software; 2005.

56. Suzuki R, Shimodaira H. Pvclust: an R package for assessing the uncertainty in hierarchical clustering. Bioinformatics. 2006;22(12):1540-2.

57. Lopes C, Pinto PA. Easy and accurate estimation of grapevine leaf area with simple mathematical models. Vitis. 2005;44(2):55-61.

58. Büttner $M$. The monosaccharide transporter(-like) gene family in Arabidopsis. FEBS Lett. 2007;581(12):2318-24.

59. Davière J-M, Achard P. Gibberellin signaling in plants. Development. 2013; 140(6):1147-51.

60. Zhiponova MK, Vanhoutte I, Boudolf V, Betti C, Dhondt S, Coppens F, et al. Brassinosteroid production and signaling differentially control cell division and expansion in the leaf. New Phytol. 2013;197(2):490-502.

61. Chung HS, Howe GA. A critical role for the TIFY motif in repression of jasmonate signaling by a stabilized splice variant of the JASMONATE ZIMdomain protein JAZ10 in Arabidopsis. Plant Cell. 2009;21(1):131-45.
62. Bessis R, Fournioux JC. Abscission zone and berry drop in grapevine. Vitis. 1992;31:9-21.

63. Malik A, Singh Z. Abscission of mango fruitlets as influenced by biosynthesis of polyamines. J Hortic Sci Biotechnol. 2003;78(5):721-7.

64. Parra-Lobato MC, Gomez-Jimenez MC. Polyamine-induced modulation of genes involved in ethylene biosynthesis and signalling pathways and nitric oxide production during olive mature fruit abscission. J Exp Bot. 2011;62(13): 4447-65.

65. Bagga S, Rochford J, Klaene Z, Kuehn GD, Phillips GC. Putrescine aminopropyltransferase is responsible for biosynthesis of spermidine, spermine, and multiple uncommon polyamines in osmotic stress-tolerant alfalfa. Plant Physiol. 1997;114(2):445-54.

66. Waduwara-Jayabahu I, Oppermann Y, Wirtz M, Hull ZT, Schoor S, Plotnikov $A N$, et al. Recycling of methylthioadenosine is essential for normal vascula development and reproduction in Arabidopsis. Plant Physiol. 2012;158(4): 1728-44.

67. Rumeau D, Peltier G, Cournac L. Chlororespiration and cyclic electron flow around PSI during photosynthesis and plant stress response. Plant Cell Environ. 2007:30(9):1041-51.

68. Butenko MA, Vie AK, Brembu T, Aalen RB, Bones AM. Plant peptides in signalling: looking for new partners. Trends Plant Sci. 2009;14(5):255-63.

69. Schaller A, Stintzi A, Graff L. Subtilases - versatile tools for protein turnover, plant development, and interactions with the environment. Physiol Plant. 2012;145(1):52-66

70. Srivastava R, Liu J-X, Howell SH. Proteolytic processing of a precursor protein for a growth-promoting peptide by a subtilisin serine protease in Arabidopsis. Plant J. 2008:56(2):219-27.

71. Galili G. New insights into the regulation and functional significance of lysine metabolism in plants. Annu Rev Plant Biol. 2002;53:27-43.

72. Joshi V, Joung J-G, Fei Z, Jander G. Interdependence of threonine, methionine and isoleucine metabolism in plants: accumulation and transcriptional regulation under abiotic stress. Amino Acids. 2010;39(4):933-47.

73. Jouhet J, Maréchal E, Block MA. Glycerolipid transfer for the building of membranes in plant cells. Prog Lipid Res. 2007:46(1):37-55.

74. Zhu J-K. Salt and drought stress signal transduction in plants. Annu Rev Plant Biol. 2002;53:247-73.

75. Araújo WL, Nunes-Nesi A, Fernie AR. Fumarate: Multiple functions of a simple metabolite. Phytochemistry. 2011;72(9):838-43.

76. Arias CL, Andreo CS, Drincovich MF, Gerrard Wheeler MC. Fumarate and cytosolic pH as modulators of the synthesis or consumption of C(4) organic acids through NADP-malic enzyme in Arabidopsis thaliana. Plant Mol Biol. 2013;81(3):297-307.

77. Sundaresan S, Philosoph-Hadas S, Riov J, Belausov E, Kochanek B, Tucker ML, et al. Abscission of flowers and floral organs is closely associated with alkalization of the cytosol in abscission zone cells. J Exp Bot. 2015;66(5):1355-68.

78. Meir S, Philosoph-Hadas S, Sundaresan S, Selvaraj KSV, Burd S, Ophir R, et al. Identification of defense-related genes newly-associated with tomato flower abscission. Plant Signal Behav. 2011;6(4):590-3.

79. Nakano T, Fujisawa M, Shima Y, Ito Y. Expression profiling of tomato preabscission pedicels provides insights into abscission zone properties including competence to respond to abscission signals. BMC Plant Biol. 2013;13:40.

80. Graeber K, Nakabayashi K, Miatton E, Leubner-Metzger G, Soppe WJ. Molecular mechanisms of seed dormancy. Plant Cell Environ. 2012;35(10):1769-86.

81. Keller MA, Piedrafita G, Ralser M. The widespread role of non-enzymatic reactions in cellular metabolism. Curr Opin Biotechnol. 2015:34C:153-61.

82. Signorelli S, Dans PD, Coitiño EL, Borsani O, Monza J. Connecting proline and $\mathrm{y}$-aminobutyric acid in stressed plants through non-enzymatic reactions. PLoS One. 2015;10(3):e0115349.

83. Guan XQ, Zhao SJ, Li DQ, Shu HR. Photoprotective function of photorespiration in several grapevine cultivars under drought stress. Photosynthetica. 2004;42(1):31-6.

84. Quiles MJ, López NI. Photoinhibition of photosystems I and II induced by exposure to high light intensity during oat plant growth. Plant Sci. 2004; 166(3):815-23.

85. Keunen E, Peshev D, Vangronsveld J, Van Den Ende W, Cuypers A. Plant sugars are crucial players in the oxidative challenge during abiotic stress: extending the traditional concept. Plant Cell Environ. 2013;36(7):1242-55.

86. Smirnoff N, Wheeler GL. Ascorbic Acid in Plants: Biosynthesis and Function. Crit Rev Biochem Mol Biol. 2000;35(4):291-314.

87. Yamaguchi S. Gibberellin metabolism and its regulation. Annu Rev Plant Biol. 2008;259:225-51. 
88. Byers $\mathrm{R}$, Carbaugh D, Presley CN, Wolf $\mathrm{T}$. The influence of low light on apple fruit abscission. J Hortic Sci Biotechnol. 1991;66(1):7-18.

89. Corelli L, Sansavini S, Ravaglia GF. Effects of shade and sorbitol on fruit growth andabscission in apple. Florence, Italy: Proceedings of the XXIII International Horticultural Congress; 1990. p. 620.

90. Zibordi M, Domingos S, Corelli Grappadelli L. Thinning apples via shading: an appraisal under field conditions. J Hortic Sci Biotechnol. 2009;ISAFRUIT S: 138-44.

91. Caspari HW, Lang A, Alspach P. Effects of girdling and leaf removal on fruit set and vegetative growth in grape. Am J Enol Vitic. 1998;49:359-66.

92. Li C, Wang Y, Huang X, Li J, Wang H, Li J. De novo assembly and characterization of fruit transcriptome in Litchi chinensis Sonn and analysis of differentially regulated genes in fruit in response to shading. BMC Genomics. 2013;14(1):552.

93. Lee $Y$, Derbyshire P, Knox JP, Hvoslef-Eide AK. Sequential cell wall transformations in response to the induction of a pedicel abscission event in Euphorbia pulcherrima (poinsettia). Plant J. 2008;54(6):993-1003.

94. Braidwood L, Breuer C, Sugimoto K. My body is a cage: mechanisms and modulation of plant cell growth. New Phytol. 2013;201(2):388-402.

95. Krasensky J, Jonak C. Drought, salt, and temperature stress-induced metabolic rearrangements and regulatory networks. J Exp Bot. 2012;63(4): 1593-608.

96. Baena-González E, Rolland F, Thevelein JM, Sheen J. A central integrator of transcription networks in plant stress and energy signalling. Nature. 2007; 448(7156):938-42.

97. Nunes C, O'Hara LE, Primavesi LF, Delatte TL, Schluepmann H, Somsen GW et al. The trehalose 6-phosphate/SnRK1 signaling pathway primes growth recovery following relief of sink limitation. Plant Physiol. 2013;162(3):1720-32.

98. Granot D, David-Schwartz R, Kelly G. Hexose kinases and their role in sugarsensing and plant development. Front Plant Sci. 2013;4:44.

99. Lastdrager J, Hanson J, Smeekens S. Sugar signals and the control of plant growth and development. J Exp Bot. 2014;65(3):799-807.

100. Gupta AK, Kaur N. Sugar signalling and gene expression in relation to carbohydrate metabolism under abiotic stresses in plants. J Biosci. 2005; 30(5):761-76.

101. Baena-González E, Sheen J. Convergent energy and stress signaling. Trends Plant Sci. 2008;13(9):474-82.

102. Alamillo JM, Díaz-Leal JL, Sánchez-Moran MV, Pineda M. Molecular analysis of ureide accumulation under drought stress in Phaseolus vulgaris L. Plant Cell Environ. 2010;33(11):1828-37.

103. Wang $\mathrm{P}$, Kong $\mathrm{C}-\mathrm{H}$, Sun $\mathrm{B}, \mathrm{Xu} \mathrm{X}-\mathrm{H}$. Distribution and function of allantoin (5ureidohydantoin) in rice grains. J Agric Food Chem. 2012;60(11):2793-8.

104. Watanabe S, Matsumoto M, Hakomori Y, Takagi H, Shimada H, Sakamoto A. The purine metabolite allantoin enhances abiotic stress tolerance through synergistic activation of abscisic acid metabolism. Plant Cell Environ. 2014; 37(4):1022-36.

105. Saika H, Okamoto M, Miyoshi K, Kushiro T, Shinoda S, Jikumaru Y, et al. Ethylene promotes submergence-induced expression of OsABA80x1, a gene that encodes ABA 8'-hydroxylase in rice. Plant Cell Physiol. 2007;48(2):287-98.

106. Zhang J, Smith D, Liu W, Chen X, Yang W. Effects of shade and drought stress on soybean hormones and yield of main-stem and branch. Afr J Biotech. 2011;10(65):14392-8.

107. Nakano T, Fujisawa M, Shima Y, Ito Y. The AP2/ERF transcription factor SIERF52 functions in flower pedicel abscission in tomato. J Exp Bot. 2014; 65(12):3111-9.

108. Harrison MA. Cross-talk between phytohormone signaling pathways under both optimal and stressful environmental conditions. In: Khan NA, Nazar R, lqbal N, Anjum N, editors. Phytohormones and abiotic stress tolerance in plants. Berlin: Springer-Verlag; 2012. p. 49-76.

109. Xin Z, Zhao Y, Zheng Z-L. Transcriptome analysis reveals specific modulation of abscisic acid signaling by ROP10 small GTPase in Arabidopsis. Plant Physiol. 2005;139(3):1350-65.

110. Yuan R, Wu Z, Kostenyuk IA, Burns JK. G-protein-coupled alpha2Aadrenoreceptor agonists differentially alter citrus leaf and fruit abscission by affecting expression of ACC synthase and ACC oxidase. J Exp Bot. 2005; 56(417):1867-75.

111. Liljegren SJ. Organ abscission: exit strategies require signals and moving traffic. Curr Opin Plant Biol. 2012;15(6):670-6

112. Mahouachi J, Iglesias DJ, Agustí M, Talon M. Delay of early fruitlet abscission by branch girdling in citrus coincides with previous increases in carbohydrate and gibberellin concentrations. Plant Growth Regul. 2009;58(1):15-23.
113. Dal Cin V, Boschetti A, Dorigoni A, Ramina A. Benzylaminopurine application on two different apple cultivars (Malus domestica) displays new and unexpected fruitlet abscission features. Ann Bot. 2007;99(6):1195-202.

114. Chen Z, Zheng Z, Huang J, Lai Z, Fan B. Biosynthesis of salicylic acid in plants. Plant Signal Behav. 2009;4(6):493-6.

115. Kunkel BN, Brooks DM. Cross talk between signaling pathways in pathogen defense. Curr Opin Plant Biol. 2002;5(4):325-31.

116. Wang X, Liu D, Li A, Sun X, Zhang R, Wu L, et al. Transcriptome analysis of tomato flower pedicel tissues reveals abscission zone-specific modulation of key meristem activity genes. PLoS One. 2013;8(2):e55238.

117. Chen M-K, Hsu W-H, Lee P-F, Thiruvengadam M, Chen H-I, Yang C-H. The MADS box gene, FOREVER YOUNG FLOWER, acts as a repressor controlling floral organ senescence and abscission in Arabidopsis. Plant J. 2011;68(1): $168-85$

\section{Submit your next manuscript to BioMed Central and we will help you at every step:}

- We accept pre-submission inquiries

- Our selector tool helps you to find the most relevant journal

- We provide round the clock customer support

- Convenient online submission

- Thorough peer review

- Inclusion in PubMed and all major indexing services

- Maximum visibility for your research

Submit your manuscript at www.biomedcentral.com/submit
) Biomed Central 